\title{
Soluble SORLA Enhances Neurite Outgrowth and Regeneration through Activation of the EGF Receptor/ERK Signaling Axis
}

\author{
Jessica Stupack, ${ }^{1}$ Xiao-Peng Xiong, ${ }^{1}$ Lu-Lin Jiang, ${ }^{1}$ Tongmei Zhang, ${ }^{1}$ Lisa Zhou, ${ }^{1}$ Alex Campos, ${ }^{2}$ \\ Barbara Ranscht, ${ }^{1}$ William Mobley, ${ }^{3}{ }^{\circledR}$ Elena B. Pasquale, ${ }^{1}{ }^{\circledR}$ Huaxi Xu, ${ }^{1}$ and ${ }^{\circledR}$ Timothy Y. Huang ${ }^{1}$ \\ ${ }^{1}$ Neuroscience Initiative, and Degenerative Diseases Program, Sanford Burnham Prebys Medical Discovery Institute, La Jolla, California 92037, \\ ${ }^{2}$ Proteomics Facility Core, Sanford Burnham Prebys Medical Discovery Institute, La Jolla, California 92037, and ${ }^{3}$ Department of Neurosciences, \\ University of California San Diego, La Jolla, California 92093
}

SORLA is a transmembrane trafficking protein associated with Alzheimer's disease risk. Although SORLA is abundantly expressed in neurons, physiological roles for SORLA remain unclear. Here, we show that cultured transgenic neurons overexpressing SORLA feature longer neurites, and accelerated neurite regeneration with wounding. Enhanced release of a soluble form of SORLA (sSORLA) is observed in transgenic mouse neurons overexpressing human SORLA, while purified sSORLA promotes neurite extension and regeneration. Phosphoproteomic analyses demonstrate enrichment of phosphoproteins related to the epidermal growth factor (EGFR)/ERK pathway in SORLA transgenic mouse hippocampus from both genders. sSORLA coprecipitates with EGFR in vitro, and sSORLA treatment increases EGFR Y1173 phosphorylation, which is involved in ERK activation in cultured neurons. Furthermore, sSORLA triggers ERK activation, whereas pharmacological EGFR or ERK inhibition reverses sSORLA-dependent enhancement of neurite outgrowth. In search for downstream ERK effectors activated by sSORLA, we identified upregulation of Fos expression in hippocampus from male mice overexpressing SORLA by RNAseq analysis. We also found that Fos is upregulated and translocates to the nucleus in an ERK-dependent manner in neurons treated with sSORLA. Together, these results demonstrate that sSORLA is an EGFR-interacting protein that activates EGFR/ ERK/Fos signaling to enhance neurite outgrowth and regeneration.

Key words: EGF receptor; ERK; neurite outgrowth; neurite regeneration; soluble SORLA; SORLA

Significance Statement

SORLA is a transmembrane trafficking protein previously known to reduce the levels of amyloid- $\beta$, which is critical in the pathogenesis of Alzheimer's disease. In addition, SORLA mutations are a risk factor for Alzheimer's disease. Interestingly, the SORLA ectodomain is cleaved into a soluble form, sSORLA, which has been shown to regulate cytoskeletal signaling pathways and cell motility in cells outside the nervous system. We show here that sSORLA binds and activates the EGF receptor to induce downstream signaling through the ERK serine/threonine kinase and the Fos transcription factor, thereby enhancing neurite outgrowth. These findings reveal a novel role for sSORLA in promoting neurite regeneration through the EGF receptor/ERK/Fos pathway, thereby demonstrating a potential neuroprotective mechanism involving SORLA.

Received Mar. 29, 2020; revised June 11, 2020; accepted June 15, 2020.

Author contributions: J.S., X.-P.X., T.Z., L.Z., A.C., and T.Y.H. performed research; J.S., X.-P.X., L.-L.J., A.C., W.M., H.X., and T.Y.H. analyzed data; L.-L.J., B.R., W.M., E.B.P., H.X., and T.Y.H. edited the paper; E.B.P., H.X., and T.Y.H. designed research; T.Y.H. contributed unpublished reagents/analytic tools; T.Y.H. wrote the first draft of the paper; T.Y.H. wrote the paper.

The authors declare no competing financial interests.

This work was supported by National Institutes of Health Grants R01 AG061875 and R01 AG044420 to T.Y.H., R01 NS046673 to H.X., and R01 NS087070 to E.B.P.; the Tanz Family Fund to H.X.; the Cure Alzheimer's Fund to H.X. and T.Y.H.; and Cancer Center Support Grant P30CA030199 to E.B.P. We thank all the members in the T.Y.H. and H.X. laboratories for critical discussions; and the Sanford Burnham Prebys Animal Resources Core, Histology Core, Proteomics Core, and Genomics Core for support.

Correspondence should be addressed to Timothy Y. Huang at thuang@sbpdiscovery.org or Huaxi Xu at xuh@sbpdiscovery.org.

https://doi.org/10.1523/JNEUROSCI.0723-20.2020

Copyright $\odot 2020$ the authors

\section{Introduction}

SORLA (sortilin-related receptor with A-type repeats) (SORL1, LR11) is a Type I transmembrane receptor previously found to be dysregulated in Alzheimer's disease (AD) in genome-wide association studies (Rogaeva et al., 2007; Lambert et al., 2013; Miyashita et al., 2013) and microarray analyses (Scherzer et al., 2004). How SORLA influences AD onset is not entirely clear. However, cumulative evidence indicates that SORLA can promote APP trafficking away from the endosome to the Golgi network (Andersen et al., 2005; Fjorback et al., 2012), or from the endosome to the cell surface (Huang et al., 2016), thus bypassing amyloidogenic $\beta$-secretase 1 cleavage in acidified endosomes. 
SORLA has also been shown to impede amyloidogenic APP cleavage by attenuating APP oligomerization (Schmidt et al., 2012) and/or by inhibiting APP/ $\beta$-secretase 1 interaction (Spoelgen et al., 2006). In addition to its role in inhibiting amyloidogenic APP processing, SORLA can also confer neuroprotection by limiting synaptotoxic EphA4 receptor activation in response to amyloid- $\beta$ ( $\mathrm{A} \beta$ ) (Fu et al., 2014; Huang et al., 2017). Despite the growing repertoire of mechanisms linking SORLA to AD pathogenesis, whether SORLA is involved in other aspects of neuroprotection remains relatively unexplored.

The SORLA ectodomain comprises an N-terminal VPS10 domain, followed by a YWTD/EGF-like domain, and multiple LDL receptor/FN3 repeats. Interestingly, SORLA can be cleaved at a site proximal to the transmembrane region by TACE/ADAM17, resulting in the release of a soluble form of the SORLA (sSORLA) extracellular region (Hermey et al., 2006). SORLA expression has been observed in various tissues, suggesting that SORLA function may not be limited to neurons or the brain. For example, SORLA expression in immune cell types (Scherzer et al., 2004), smooth muscle cells (Zhu et al., 2004; Jiang et al., 2006), and adipose tissue (Schmidt et al., 2016) suggests potential roles for SORLA in atherosclerosis and insulin-related obesity. SORLA expression in white adipose tissue positively correlates with body mass index and enhances cell surface distribution of the insulin receptor to potentially promote adiposity (Schmidt et al., 2016). Serum levels of sSORLA also positively correlate with body mass index, and sSORLA was found to suppress thermogenic metabolism in brown adipose tissue through inhibition of the TGF $\beta$ /SMAD pathway (Whittle et al., 2015). sSORLA can also stimulate smooth muscle cell migration (Zhu et al., 2004; Jiang et al., 2006) and potentially mediates medial to intimal smooth muscle cell migration, which may be critical to atherosclerotic plaque formation (Jiang et al., 2008). Despite the potential roles of sSORLA described in peripheral systems and previous studies suggesting a protective role for SORLA in AD (Andersen et al., 2005; Spoelgen et al., 2006; Schmidt et al., 2012; Caglayan et al., 2014; Huang et al., 2016, 2017), physiological functions for SORLA and whether SORLA ectodomain shedding from neurons can confer neuroprotective effects remains unclear.

Here, we demonstrate a previously undescribed role for SORLA in enhancing neurite outgrowth and regeneration. Transgenic neurons overexpressing SORLA (SORLA TG) and neurons exposed to the purified sSORLA ectodomain show increased neurite extension and accelerated neurite regeneration following wound injury in culture. Analysis of global changes in the SORLA TG phosphoproteome from mouse hippocampus reveals enrichment of phosphopeptides associated with the EGFR/ERK MAPK signaling pathway, suggesting that sSORLA upregulation promotes EGFR activation and downstream ERK signaling. Indeed, exposure to sSORLA or transgenic SORLA/ sSORLA overexpression similarly promotes ERK activation, whereas SSORLA-dependent neurite regeneration is abrogated by EGFR or ERK inhibition. Together, our results implicate sSORLA as an EGFR binding partner that drives neurite outgrowth and regeneration through ERK.

\section{Materials and Methods}

Mouse lines. The SORLA-Rosa26 (SORLA TG in the BALB/c background) mouse line overexpressing SORLA through a CMV/ $\beta$-actin promoter element was previously established (Caglayan et al., 2014). SORLA KO mice were obtained from Thomas Willnow (Max Delbrueck Center for Molecular Medicine, Berlin) (Andersen et al., 2005). All procedures were compliant with the National Research Council's Guide for the care and use of laboratory animals, and approved by the Sanford Burnham Prebys Medical Discovery Institute Institutional Animal Care Use Committee. Mice were housed 2-4 animals per cage under standard conditions, maintaining a $12 \mathrm{~h}$ light/dark cycle and consistent access to animal water and chow.

Antibodies, constructs, and protein purification. Primary antibodies were all purchased from commercial sources: SORLA (LR11; BD Biosciences), FLAG M2 (Stratagene/Sigma Millipore), $\beta$-actin, $\beta$ IIItubulin (Tuj1) and $\alpha$-tubulin (Sigma Millipore), EGFR (CST 2232S), pEGFR (Y1173, CST 4407S), p-ERKPhospho p44/42 MAPK (Erk1/2) (Thr202/Tyr204) Antibody CST 9101S), ERK1/2 (CST 9102S P44/42), Fos (ab190289 Abcam), EphA4 (EphA4 (Santa Cruz Biotechnology, S20), APP (Sigma Millipore, MAB348), soluble APP $(\operatorname{sAPP} \alpha)(B 436$ monoclonal antibody), TrkB (R\&D Systems, AF1494, human/mouse/rat TrkB antibody), Erbb2 (R\&D Systems, AF5176), and IR (anti-insulin receptor $\alpha$ antibody, Abcam, ab5500). Secondary antibodies include the following: anti-rabbit IgG HRP-linked antibody (CST, 7074S), antimouse IgG HRP-linked antibody (CST, 7076S), Alexa-568 phalloidin (Invitrogen, catalog \#A12380), Alexa-488 donkey anti-rabbit IgG (Thermo Fisher Scientific, catalog \#A21206), donkey anti-mouse IgG Alexa-488 (Thermo Fisher Scientific, catalog \#A21202), and donkey anti-rabbit IgG Alexa-568 (Thermo Fisher Scientific, catalog \#A10042).

A sSORLA overexpression construct was generated by insertion of an XbaI site at residue EMMLV (upstream of the predicted transmembrane region) by PCR-based site-directed mutagenesis of a pcDNA3 SORLA template, where a FLAGhis ${ }_{6}$ sequence with XbaI overhangs was inserted in-frame of the XbaI site. Plasmids were transfected into HEK293T cells overnight using Turbofect reagent (Thermo Fisher Scientific); cells then were washed with $1 \times$ PBS and incubated in serumfree DMEM (supplemented with glutamine/penicillin/streptomycin) for $2 \mathrm{~d}$. Media was then collected and centrifuged at $500 \times g$ to remove any cells, and incubated with NTA-agarose in the presence of $10 \mathrm{~mm}$ imidazole with constant agitation for $4 \mathrm{~h}$ to overnight. Beads were then collected by centrifugation, washed thoroughly 4 times in $10 \mathrm{~mm}$ Tris, $\mathrm{pH}$ 8.0, $0.5 \mathrm{M} \mathrm{NaCl} / 20 \mathrm{~mm}$ imidazole, and the his ${ }_{6}$-tagged protein was eluted in $0.3 \mathrm{~m}$ imidazole in $10 \mathrm{~mm}$ Tris, $\mathrm{pH} 8.0,0.5 \mathrm{~m} \mathrm{NaCl}$. sSORLAFLAGhis 6 was dialyzed in $1 \times$ PBS overnight, and quantified by silver stain gel staining using a BSA protein standard. Glycerol was added to purified SSORLA, and the samples were frozen in aliquots at $-80^{\circ} \mathrm{C}$.

Primary neuronal culture. Embryos were harvested from pregnant mice at embryonic day 17 (EXVII)-EXVIII from SORLA TG $\mathrm{x}$ WT $(\mathrm{BALB} / \mathrm{c})$ crosses. Primary hippocampal and cortical neurons were isolated by microdissection from the cerebral cortex and hippocampus using a stereomicroscope. Tissue was digested with trypsin and DNase 1 for $30 \mathrm{~min}$ at $37^{\circ} \mathrm{C}$, followed by trituration in DMEM with penicillin/ streptomycin and HEPES (Thermo Fisher Scientific, catalog \#15630080). Tissue from embryos used to generate SORLA TG neurons was also collected and processed for genotyping analysis for SORLA TG neurons using the MyTaq DNA extraction/PCR genotyping system (Bioline). Individual neuronal genotypes were maintained separately on poly-D-lysine-coated coverslips and plates. Neurons were cultured in Neurobasal Medium supplemented with B27, glutamine, and penicillin/streptomycin, where half of the media was replaced every $2-3 \mathrm{~d}$.

Laceration injury. Cortical neurons were cultured on poly-D-lysinecoated $12 \mathrm{~mm}$ coverslips in 24-well culture plates until DIV6. Neurites were then subjected to laceration injury (mechanical wounding) by dragging a $10 \mu \mathrm{l}$ pipette tip steadily across the coverslip. The cultures were then treated with recombinant sSORLA (typically at $150 \mathrm{ng} / \mathrm{ml}$, unless otherwise indicated) or vehicle, and neurites were allowed to recover for $16 \mathrm{~h}$ before fixation and staining.

Immunocytochemistry. Immunocytochemistry was performed on primary cultured hippocampal or cortical neurons to image, characterize, and quantify neurite length, and nuclear Fos labeling. Neurons on glass coverslips were washed with PBS, fixed with $4 \%$ PFA, and permeabilized with $0.1 \%$ Triton X-100 in PBS. After blocking in 3\% BSA in PBS, primary antibodies were diluted in $1 \%$ BSA and used to probe neurons grown on coverslips for $1 \mathrm{~h}$ at room temperature with protection from light. Primary antibody incubation (or F-actin staining with 
phalloidin) was followed by staining with AlexaFluor-conjugated secondary antibodies (Invitrogen), and DAPI staining. Stained coverslips were mounted on glass slides using mounting media containing an antifade reagent. Images were acquired by epifluorescence imaging using a deconvolution microscope (Carl Zeiss), and images were processed using Slidebook 6.0 software (Intelligent Imaging Innovations), or using an LSM 880 confocal microscope (Carl Zeiss). Confocal and epifluorescence image analyses were performed using National Institutes of Health Image J (Fiji).

Measurement of neurite and axon length, and quantification of neurite number per neuron. Neurites (dendrites and axons) per neuron were scored as the number of projections with active growth cones extending from neuronal bodies. Axons were defined by dendritic extensions, which were at least twice the length of all other neurites, and having a constant thickness throughout the length of the axon as described previously (Richter et al., 2007). Neurite length in images was measured and quantified using the freehand line measurement on Fiji. For neurite regeneration measurements, neurite length originating from the edge of the laceration border to the tips of individual neurites was traced and quantified.

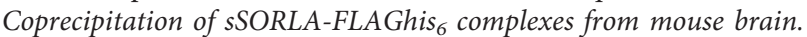
sSORLA (500 ng) was immobilized to $25 \mu$ l of $50 \%$ slurry M2 beads overnight. Solution was washed with wash/lysis buffer (10 mM Tris, $\mathrm{pH}$ 8.0, $0.5 \mathrm{M} \mathrm{NaCl}) 4 \times$ before confirmation of sSORLA binding via silver stain (at least $50 \mathrm{ng}$ sSORLA was detected in duplicate pulldown reactions). Immobilized sSORLA was then incubated with $0.5 \mathrm{mg}$ of neuronal lysate, rocking at 4 degrees for $4 \mathrm{~h}$. Bound components were gently washed twice in PBS (e.g., $0.1 \mathrm{~m}$ phosphate, $0.15 \mathrm{~m} \mathrm{NaCl}, \mathrm{pH} 7.2$ ) and crosslinked using $1 \mathrm{~mm}$ dithiobis(succinimidyl propionate) crosslinker solution for $30 \mathrm{~min}$ at room temperature, and quenched with $20 \mathrm{~mm}$ Tris, pH 7.5, for $15 \mathrm{~min}$. Beads were then washed and boiled in SDS loading buffer with $\beta$-mercaptoethanol for $10 \mathrm{~min}$ before SDS-PAGE and immunoblot analysis; 20 ng of lysate was included as input.

Western blotting. Samples were lysed in NP40 buffer (1\% Non-Idet P40 in PBS, supplemented with protease and phosphatase inhibitors) and SDS sample buffer was added. Samples were resolved by SDS-PAGE using 4\%-20\% Tris/glycine gels, transferred onto nitrocellulose blotting membrane using a Bio-Rad electroblot system, and blocked in 5\% dry milk in PBS. Membranes were probed with primary and secondary antibodies, and developed using ECL or ECL plus (Thermo Fisher Scientific).

Phosphoproteome analysis. Brain cortical tissues from WT and SORLA TG littermates of mixed gender were lysed in $8 \mathrm{~m}$ urea, $50 \mathrm{~mm}$ ammonium bicarbonate with Benzonase, and extracted proteins were centrifuged at $14,000 \times g$ for $10 \mathrm{~min}$ to remove cellular debris. Supernatant protein concentration was determined using a bicinchoninic acid protein assay (Thermo Fisher Scientific). Disulfide bridges were reduced by incubation with $5 \mathrm{~mm}$ tris (2-carboxyethyl)phosphine at $30^{\circ} \mathrm{C}$ for $60 \mathrm{~min}$, and cysteines were subsequently alkylated with $15 \mathrm{~mm}$ iodoacetamide in the dark at room temperature for $30 \mathrm{~min}$. Urea was then diluted to $1 \mathrm{~m}$ urea using $50 \mathrm{~mm}$ ammonium bicarbonate, and proteins were subjected to overnight digestion with mass spectrometry grade Trypsin/Lys-C mix (Promega). Following digestion, samples were acidified with formic acid and subsequently peptides were desalted using AssayMap C18 cartridges mounted on an Agilent AssayMap BRAVO liquid handling system.

Phosphopeptides were enriched with Fe(III)-NTA cartridges (Agilent Technologies) in an automated fashion using the AssayMAP Bravo Platform (Agilent Technologies). Briefly, cartridges were primed with $250 \mu \mathrm{l}$ of $0.1 \%$ trifluoroacetic acid in acetonitrile (ACN) and equilibrated with IMAC loading buffer ( $80 \% \mathrm{ACN}, 0.1 \%$ trifluoroacetic acid). Dried samples were reconstituted in IMAC loading buffer and loaded onto the cartridge, which was then washed with IMAC loading buffer to remove background. Finally, phosphorylated peptides were eluted with $1 \%$ ammonia directly into formic acid. Finally, the samples were dried down in a SpeedVac concentrator. Before LC-MS/MS analysis, sample peptide amount was determined using a Nanodrop spectrophometer (Thermo Fisher Scientific).

Dried phosphopeptide-enriched samples were reconstituted with $2 \%$ ACN/0.1\% FA and analyzed by LC-MS/MS using a nanoACQUITY system (Waters) coupled to an Orbitrap Fusion Lumos mass spectrometer (Thermo Fisher Scientific). Peptides were separated using an analytical
C18 Acclaim PepMap column $(75 \mu \mathrm{m} \times 250 \mathrm{~mm}, 2 \mu \mathrm{m}$ particles; Thermo Fisher Scientific) using a 74 min gradient, at flow rate of $300 \mu \mathrm{l} /$ min, consisting of the following: $1 \%$ to $5 \% \mathrm{~B}$ in $1 \mathrm{~min}, 5 \%$ to $18 \% \mathrm{~B}$ in $44 \mathrm{~min}, 18 \%$ to $27 \% \mathrm{~B}$ in $28 \mathrm{~min}, 27 \%$ to $38 \% \mathrm{~B}$ in $2 \mathrm{~min}$, and $38 \%$ to $80 \% \mathrm{~B}$ in $2 \min (\mathrm{A}=\mathrm{FA} 0.1 \%$; $\mathrm{B}=100 \% \mathrm{ACN}: 0.1 \% \mathrm{FA})$. The mass spectrometer was operated in positive data-dependent acquisition mode. MS1 (mass spectrometry precursor intensity at maximum peak elution for quantitation) spectra were measured in the Orbitrap with a resolution of 120000 (AGC target: 1e6; maximum injection time: $50 \mathrm{~ms}$; mass range: from 350 to $1200 \mathrm{~m} / \mathrm{z}$ ). The instrument was set to run in top speed mode with $3 \mathrm{~s}$ cycles for the survey and the MS/MS scans. After a survey scan, precursors were isolated in the quadrupole (isolation window: $0.7 \mathrm{~m} / \mathrm{z}$; charge state: $+2-6$; collision energy: $30 \%)$, and resulting fragments were detected in the ion trap cell with a rapid scan (AGC target for MS/MS: 2e4; maximum injection time: $35 \mathrm{~ms}$ ). The dynamic exclusion was set to $20 \mathrm{~s}$ with a $10 \mathrm{ppm}$ mass tolerance around the precursor and its isotopes.

All mass spectra were analyzed with MaxQuant software version 1.5.5.1. MS/MS spectra were searched against the Mus musculus Uniprot protein sequence database (downloaded in January 2019) and GPM cRAP sequences (commonly known protein contaminants). Precursor mass tolerance was set to $20 \mathrm{ppm}$ and $4.5 \mathrm{ppm}$ for the first search where initial mass recalibration was completed and for the main search, respectively. Product ions were searched with a mass tolerance $0.5 \mathrm{Da}$. The maximum precursor ion charge state used for searching was 7. Carbamidomethylation of cysteine was searched as a fixed modification, while oxidation of methionine and acetylation of protein $\mathrm{N}$-terminal, and phosphorylation of serine, threonine, and tyrosine were searched as variable modifications. Enzyme was set to trypsin in a specific mode, and a maximum of two missed cleavages was allowed for searching. The target-decoy-based false discovery rate filter for spectrum and protein identification was set to $1 \%$.

Statistical analysis of phosphopeptide data were conducted using inhouse R script (version 3.5.1, 64 bit), including R Bioconductor packages, such as limma and MSstats. First, phosphopeptide intensities were $\log _{2^{-}}$ transformed and Loess-normalized across samples to account for systematic errors. Testing for differential abundance was performed using MSstats bioconductor package based on a linear mixed-effects model. Imputed $\log _{2} \mathrm{FC}$ and $p$ values were calculated for phosphopeptides missing in one of the conditions. First, imputed $\log _{2} \mathrm{FC}$ was calculated as the average $\log _{2}$ intensity of the phosphopeptide across samples in the condition it is detected, divided by 3.3. Imputed $p$ value was calculated by dividing 0.05 by the number of replicates within the detected condition of the phosphopeptide identified. Phosphoproteomic data included in this study has been deposited in the ProteomeXchange database (http://www. proteomexchange.org/), under reference no. PXD019108.

RNAseq analysis and $q R T-P C R$ validation. For RNAseq analysis of WT and SORLA TG brain, hippocampal tissue from 3-month-old male mice ( $n=2 \mathrm{WT}, n=3$ SORLA TG) was dissected and washed in cold PBS. Tissue was homogenized and Dounced in TRIzol Reagent (Thermo Fisher Scientific, catalog \#15596026) and stored at $-80^{\circ} \mathrm{C}$ for RNA extraction. Total RNA was purified with Trizol reagent combined with chloroform, following the manufacturer's recommendations. RNA was quantified using a NanoDrop spectrophotometer, and RNA integrity numbers were assessed using an Agilent TapeStation 4200 system; all samples used for subsequent analysis showed a minimum RNA integrity number of 9 . For each replicate, 250 ng of purified total RNA was submitted to Novogene for mRNA RNAseq library preparation and sequence analysis with paired-end reads. A minimum sequence depth of $50 \mathrm{M}$ with $150 \mathrm{bp}$ paired-end reads was characterized for each sample. Reads were aligned to the Mus musculus genome (mm10) using STAR aligner (https://code.google.com/p/rna-star/) under default settings. Differential transcript expression was characterized using DESeq2 $R$ package. Cutoffs for significantly altered differentially expressed genes (DEGs) were set to $p<0.05$ with a $\log _{2}$ fold cutoff of \pm 0.3 . RNAseq data included in this study has been deposited in the Gene Expression Omnibus database under the accession no. GSE150257.

DEGs in SORLA TG versus WT $\left(p<0.05, \log _{2}\right.$ fold change $<-0.3$ or $>0.3$ ) were subjected to KEGG pathway analysis and Gene ontology 
A
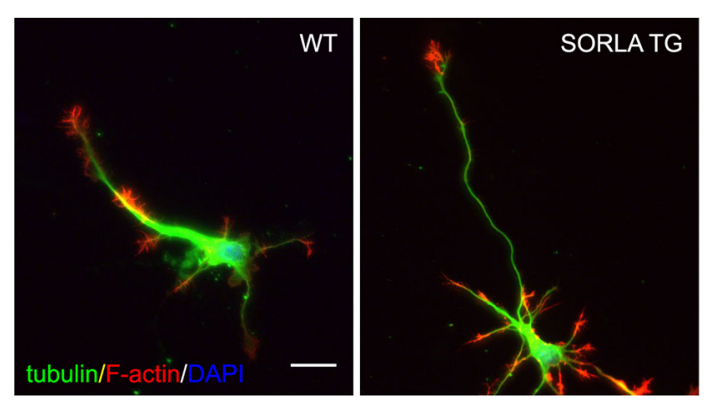

$\mathbf{E}$

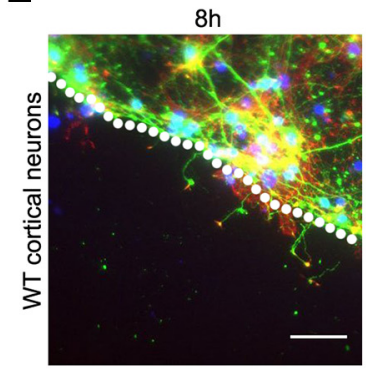

$\mathbf{F}$
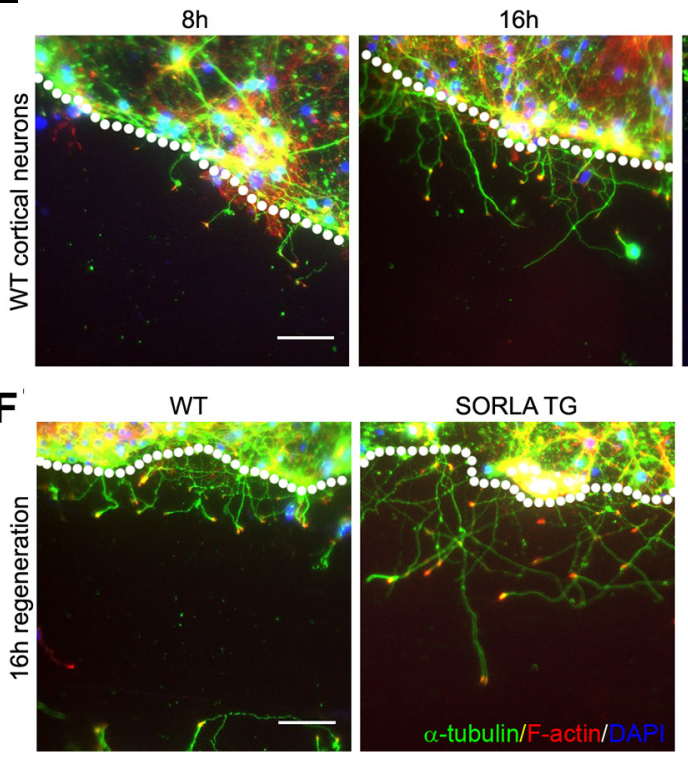

B

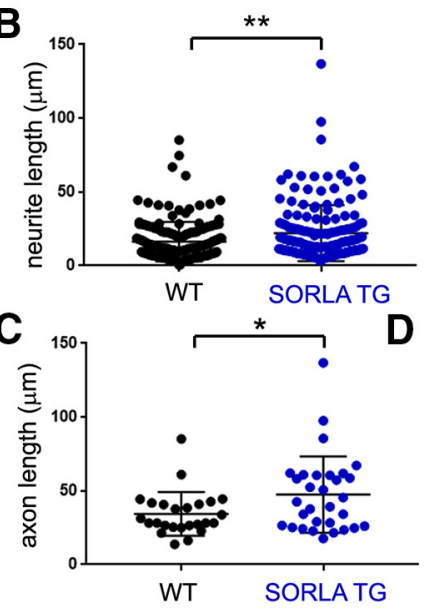

$22 \mathrm{~h}$
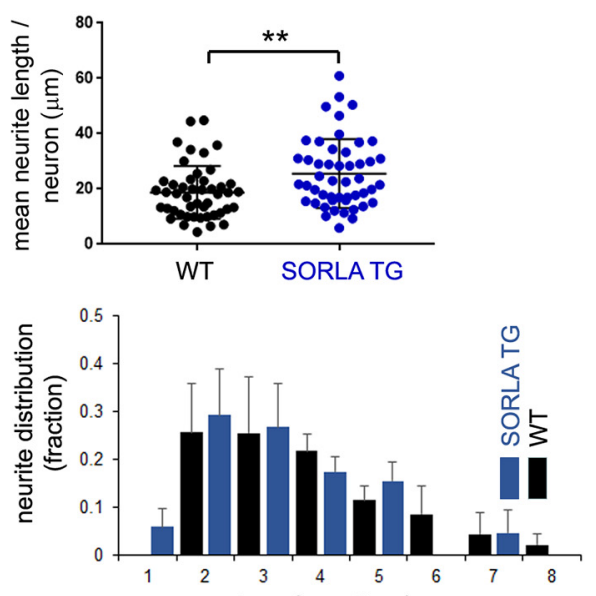

number of neurites / neuron

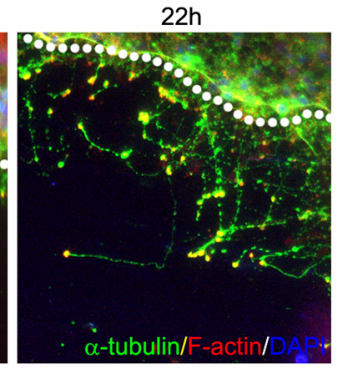

G
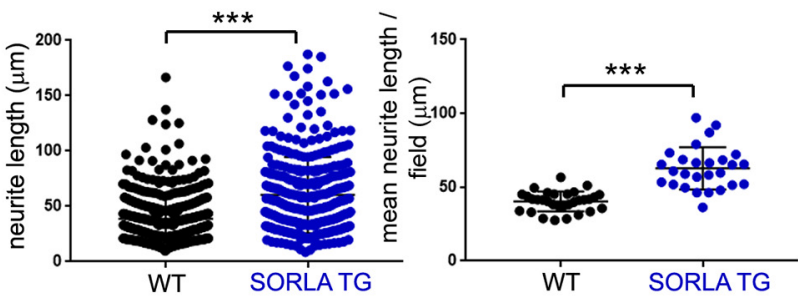

Figure 1. SORLA overexpression enhances neurite outgrowth and regeneration in cultured neurons. $A$, WT and SORLA TG hippocampal neurons were fixed and stained for tubulin (green), Factin (phalloidin, red), and DAPI (blue) at DIV3, and neuronal morphology was examined by fluorescence microscopy. Scale bar, $10 \mu \mathrm{m}$. $\boldsymbol{B}$, Neurite length in WT and SORLA TG hippocampal neurons was scored in all neurites imaged (left; WT, 173 neurites, 43 neurons; SORLA TG, 164 neurites, 49 neurons; 3 independent dissections/cultures per treatment; $p=0.0013, t$ test), or averaged per neuron (right; WT, 43 neurons; SORLA TG, 49 neurons; 6 coverslips, 4 independent dissections/cultures per genotype; $p=0.0037, t$ test). C, Axon length in WT compared with SORLA TG polarized hippocampal neurons (WT, 25 axons; SORLA TG, 31 axons; 8 coverslips, 3 independent dissections/cultures per genotype; $p=0.0283, t$ test). $\boldsymbol{D}$, The number of neurites per neuron were scored in WT (black) and SORLA TG (blue) neurons, and calculated as a fraction of the total neurons scored for each genotype (WT, 116 neurites, 31 neurons; SORLA TG, 127 neurites, 29 neurons; 6 coverslips, 3 independent dissections/cultures per genotype). E, WT and SORLA TG cortical neurons were wounded at DIV7, and neurite infiltration into the wound area was visualized by immunostaining (tubulin [green], F-actin [red], DAPI [blue])/fluorescence imaging at 8,16 , and $22 \mathrm{~h}$ following injury (h). Scale bar, $30 \mu \mathrm{m}$. $\boldsymbol{F}$, Cortical neurons on coverslips were subjected to scratch injury at DIV7, and neurite penetration into the wound area was visualized by immunostaining (tubulin [green], F-actin [red], DAPI [blue])/fluorescence imaging at $16 \mathrm{~h}$ following injury. Scale bar, $30 \mu \mathrm{m}$. G, Neurite lengths were quantified from all neurons imaged in $\boldsymbol{F}$ (left; WT, 422 neurites, 31 images; SORLA TG, 352 neurites, 29 images; 9 coverslips from 3 independent dissections per genotype; $p<0.0001, t$ test), or quantified as averages per image field (right; WT $n=31$ imaging fields; SORLA TG, $n=27$ imaging fields; 9 coverslips from 3 independent dissections per genotype; $p<0.0001, t$ test). $\boldsymbol{B}, \mathbf{C}, \mathbf{G}$, Data are mean \pm SD. D, Data are mean \pm SE. ${ }^{*} p<0.05 ;{ }^{* *} p<0.01 ;{ }^{* * *} p<0.0001 ;$ Student's $t$ test.

(GO) profiles of DEGs were determined using DAVID (database for annotation, visualization and integrated discovery) (Huang da et al., 2009a,b). cDNA from mouse hippocampal tissue samples was generated from mRNA using the iScript cDNA synthesis kit from (Bio-Rad) according to specified protocols from the manufacturer using Powerup SYBR Green Master Mix (Thermo Fisher Scientific) together with the Bio-Rad CFX detection system according to the manufacturer's instructions. Expression values for each target gene was normalized to the internal actin and GAPDH controls. Primer sequences used for qRT-PCR analysis are as follows: SCN1B: forward 5'-GGATTCCGATACCG AGGCTG; reverse 5'-GGTCCACTCCGTGAAGGTTT; NREP: forward 5'-GGTGTCGGTACTTTGTTTCCTGG; reverse 5'-CTCACACTCTT GGTAGCATCCAC; GPNMB: forward 5'-GCTGGTCTTCGGATGAA AATGA; reverse 5' -CCACCAAGGTGATATTGGAACCC; LPAR: forward 5'-AGCCATGAACGAACAACAGTC; reverse 5'-CATGATGA
ACACGCAAACAGTG; KIF26B: forward 5'-TCGGTAGCCGGAAAT AAAGAGA; reverse 5' -CGACTCCTCGTAAGCCTTGC; IRS1: forward 5'-CGATGGCTTCTCAGACGTG; reverse 5' -CAGCCCGCTTGTTGA TGTTG; FOS: forward 5'-CGGGTTTCAACGCCGACTA; reverse $5^{\prime}$ TTGGCACTAGAGACGGACAGA; SEMA3D: forward 5'-CTGTAT CCCCTTTTTGGGTTCAT; reverse 5'-AACCAGACTGAGGAAGAC; SEMA3F: forward 5'-CATCTGCCTCAACGATGACG; reverse $5^{\prime}$ AGAGCCTGAAGAGGTAAAGAC; UNC5B: forward 5'-CTGGGG ACCGGGAAAGAA; reverse 5' -CTGATGGGTAGGAGTCTGGG.

Experimental design and statistical analyses. For all experiments using cultured neurons, cultures from a minimum of two independent dissections were used for analyses. Measurements quantifying neurite length or biochemical analyses were pooled from independent experiments as indicated. All statistical analyses used in this study evaluated significant differences between two groups using nonpaired $t$ tests with 


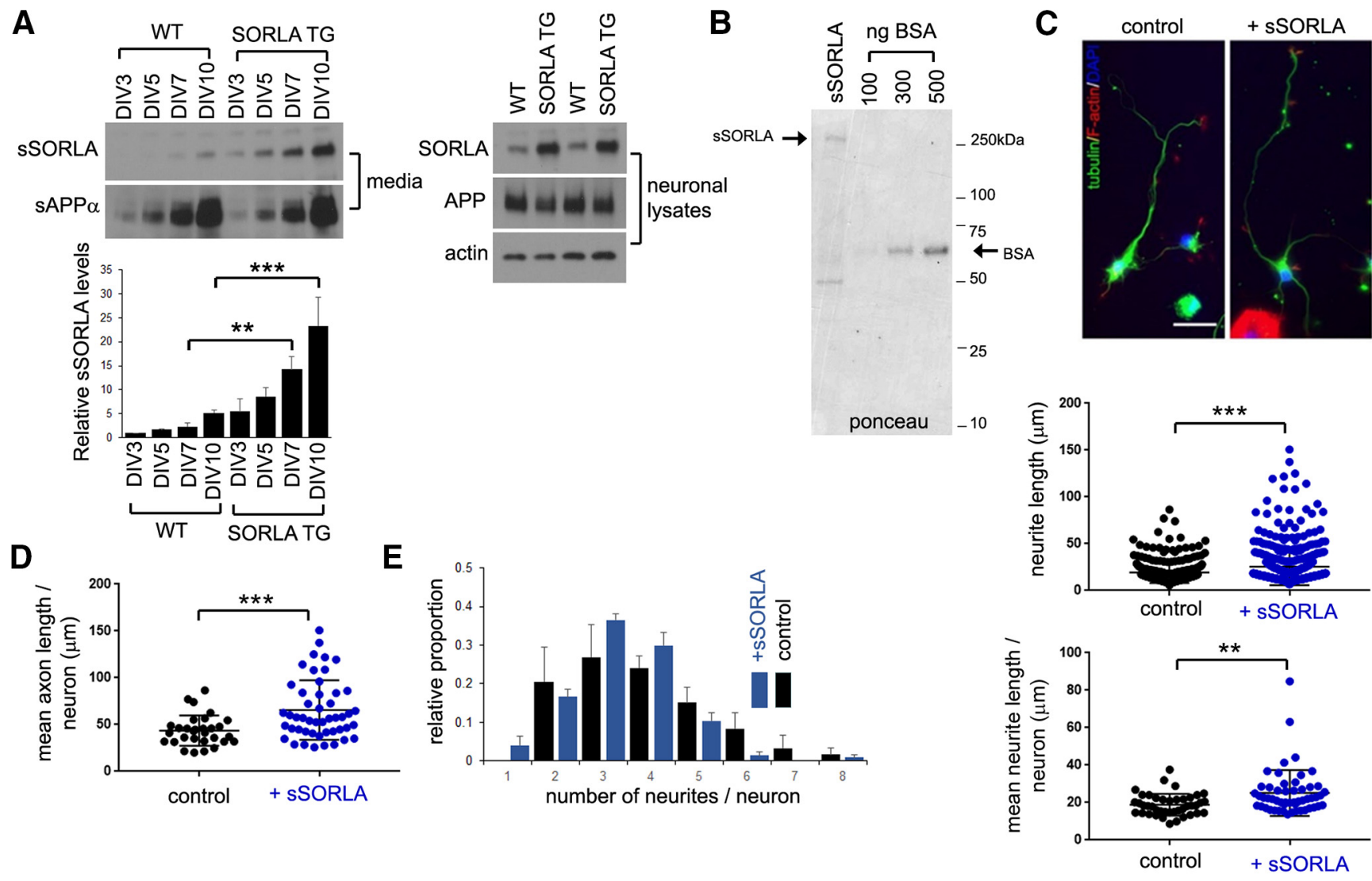

Figure 2. An sSORLA fragment enhances neurite outgrowth. $A$, Conditioned media from cultured WT or SORLA TG cortical neurons were sampled from DIV3 to DIV10 and immunoblotted for SSORLA or SAPP $\alpha$ as indicated. Neuronal cell lysates were also immunoblotted for full-length SORLA or APP as indicated (right). Relative SSORLA levels were quantified by densitometry (bottom, $n=3$ experiments). sSORLA levels from DIV3 neurons were set to $1.0\left(F_{(3,16)}=5.307\right.$, DIV7 WT vs TG, $p=0.0036$; DIV10 WT vs TG, $p<0.0001$, two way ANOVA with Tukey's multiple comparisons). $\boldsymbol{B}$, SSORLA (sSORLA-FLAGhis 6 ) expressed in HEK293T cells was purified from conditioned medium using nickel NTA-agarose, and subjected to Ponceau $S$ staining following transfer onto nitrocellulose membranes. BSA was stained to determine the concentration of purified sSORLA. C, WT hippocampal neurons at DIV2 were incubated overnight with $150 \mathrm{ng} / \mathrm{ml}$ purified sSORLA, and stained for tubulin (green), F-actin (red), and DAPI. Scale bar, $10 \mu \mathrm{m}$. Graphs represent quantification of the length of all neurites in WT control (black) and SSORLA (blue) treated hippocampal neurons (top) or the neurite length averaged per neuron (bottom) (control, 164 neurites, 46 neurons; sSORLA, 182 neurites, 55 neurons; 6 coverslips, 3 independent dissections/cultures per treatment; top: $p<0.0001$; bottom: $p=0.0027, t$ test). $\boldsymbol{D}$, Axon length was measured in hippocampal neurons identified with axonal extensions in control and SSORLA-treated WT neurons (control, 30 axons/neurons; SSORLA, 48 axons/neurons; 6 coverslips, 3 independent dissections/cultures per treatment) ( $p=0.0008, t$ test). $\boldsymbol{E}$, The number of neurites was scored in control and sSORLA-treated WT hippocampal neurons (control, 164 neurites scored in 46 neurons; SSORLA, 182 neurites scored in 55 neurons; 6 coverslips, 3 independent dissections/cultures per treatment). $A, E$, Data are mean \pm SE. C, D, Data are mean \pm SD. C, D, ${ }^{* *} p<0.01 ;{ }^{* * *} p<0.001$; Student's $t$ test; $A$, Two-way ANOVA followed by Tukey's multiple comparisons.

equal variance, or ANOVA from a minimum of three groups with post hoc comparisons to evaluate significant differences between groups. Significance was calculated using GraphPad Prism 7.03. Details regarding statistical tests for individual experiments are described in the corresponding figure legends.

EGFR and ERK inhibitor treatment. For experiments involving EGFR inhibition (AG-1478) (Sigma Millipore) and analysis by immunoblotting, cortical neurons were treated with $100 \mu \mathrm{M}$ AG-1478 for $3 \mathrm{~h}$, and subsequently with sSORLA $(150 \mathrm{ng} / \mathrm{ml})$ or EGF for $30 \mathrm{~min}$. For and ERK inhibition experiments and analysis by immunoblotting, cortical neurons were exposed to the MEK inhibitor (PD98059, $100 \mu \mathrm{M}$ ) (Invivogen) for $24 \mathrm{~h}$, followed by treatment with sSORLA $(150 \mathrm{ng} / \mathrm{ml})$ for $30 \mathrm{~min}$. For neurite regeneration assays, AG-1478 or PD98059 was added to cortical neurons concurrently with EGF or sSORLA immediately following laceration injury, and neurites were allowed to regenerate for $16 \mathrm{~h}$. Neurons were then fixed and imaged, and quantified for neurite length.

\section{Results}

SORLA upregulation enhances neurite outgrowth

Although transgenic SORLA overexpression has been shown to be neuroprotective by limiting amyloidogenic APP processing
(Andersen et al., 2005) and $\mathrm{A} \beta$-mediated synaptotoxicity (Huang et al., 2017), a protective role for SORLA through enhancement of neural regeneration has not been established. We therefore compared morphologic differences in WT and SORLA-overexpressing (SORLA TG) neurons. Hippocampal SORLA TG neurons cultured for DIV3 showed significantly longer neurites compared with WT neurons (Fig. $1 A-C$ ). We observed increased total neurite length in all neurons scored as well as increased mean neurite length averaged per neuron (Fig. $1 B)$. Axons in polarized SORLA TG neurons were also longer (Fig. 1C). However, we did not observe any significant differences in the distribution of neurite numbers (Fig. 1D). This suggests that SORLA overexpression enhances neurite length with little or no influence on overall neurite number.

To further characterize the effects of SORLA overexpression on neurite regeneration, we compared the extension of cortical WT and SORLA TG neurons into a wound area generated at DIV7 by mechanical injury. We found that the length of SORLA TG neurites infiltrating into the wound area at 16 and $22 \mathrm{~h}$ following injury was significantly greater compared with the length of WT neurites (Fig. $1 E-G$ ). Together, these results indicate that 
A

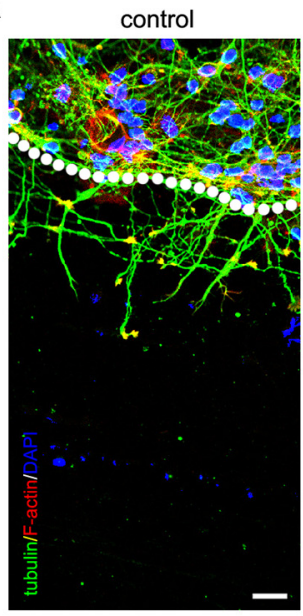

$50 \mathrm{ng} / \mathrm{ml}$ sSORLA

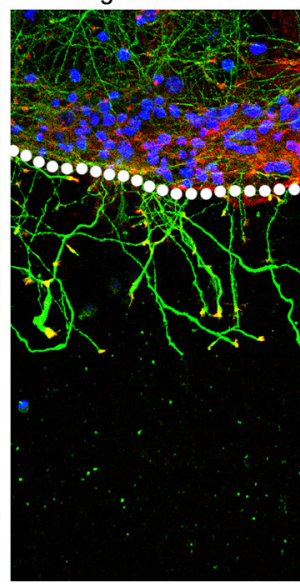

$100 \mathrm{ng} / \mathrm{ml}$ sSORLA

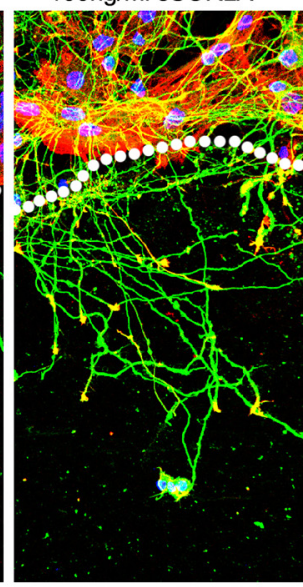

$150 \mathrm{ng} / \mathrm{ml}$ sSORLA

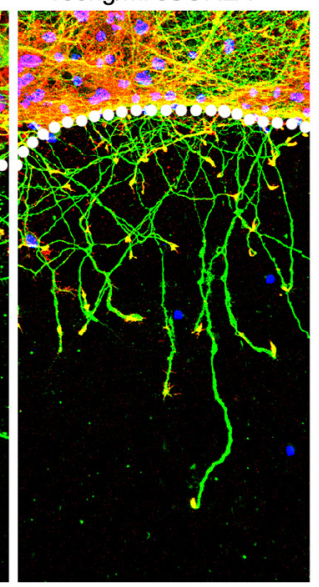

B
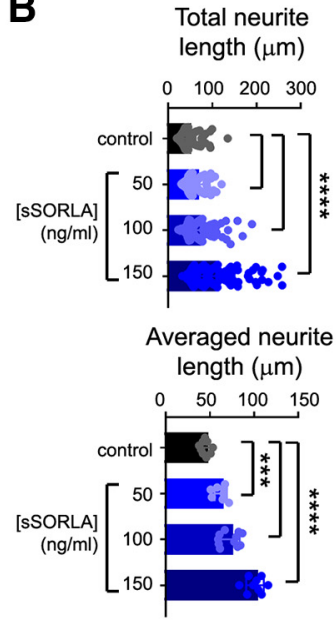

C
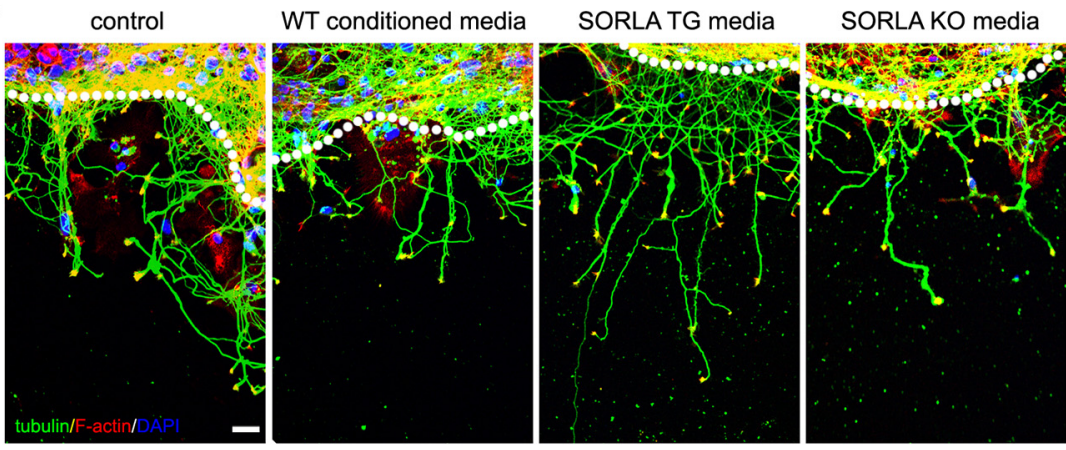

D
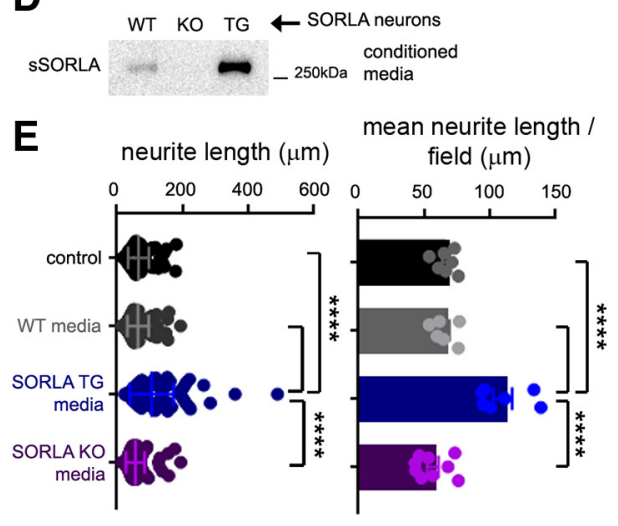

Figure 3. SSORLA enhances neurite outgrowth following injury in vitro. $\boldsymbol{A}$, WT cortical neurons were subjected to mechanical wound injury at DIV7 and treated with purified SSORLA at the concentrations indicated for $16 \mathrm{~h}$. Neurons were then fixed and stained for tubulin (green), F-actin (red), or DAPI. Scale bar, $20 \mu \mathrm{m}$. B, Quantified total (top) and averaged neurite length/ imaged field (bottom) following injury in SSORLA-treated and WT neurons (control, 165 neurites $/ 9$ images; $50 \mathrm{ng} / \mathrm{ml}, 153$ neurites in 10 images; $100 \mathrm{ng} / \mathrm{ml}$, 172 neurites in 11 images; $150 \mathrm{ng} /$ ml sSORLA, 166 neurites in 9 images; 3 coverslips from two independent dissections $/ 3$ cultures per treatment) (top: $F_{(3,610)}=107.9$, control vs $50 \mathrm{ng} / \mathrm{ml}, \mathrm{vs} 100 \mathrm{ng} / \mathrm{ml}$, and vs $150 \mathrm{ng} / \mathrm{ml}$ sSORLA, $p<0.0001$; bottom: $F_{(3,34)}=64.93$, control vs $50 \mathrm{ng} / \mathrm{ml}, p=0.0009$, control vs $100 \mathrm{ng} / \mathrm{ml}$ and $150 \mathrm{ng} / \mathrm{ml}$ sSORLA, $p<0.0001$, one-way ANOVA with Tukey's multiple comparisons). C, WT cortical neurons at DIV 7 were subjected to scratch injury and treated with neurobasal/B27 medium (control), or conditioned medium from WT, SORLA TG, or SORLA K0 neurons for 16 h, and stained and imaged as in $A$. Scale bar, $20 \mu \mathrm{m}$. D. Neurobasal media conditioned for $2 \mathrm{~d}$ from DIV7 WT, SORLA KO, or SORLA TG neurons were immunoblotted for sSORLA. $\boldsymbol{E}$, Lengths from all neurites (left) or averaged per imaged field (right) were quantified from images in experiments as shown in $\boldsymbol{D}$. The data were obtained from control, 142 neurites in 9 images; WT medium, 149 neurites in 9 images; SORLA TG medium, 139 neurites in 9 images; SORLA KO medium, 147 neurites in 9 images; 3 coverslips from two independent dissections/3 cultures per treatment (left: $F_{(3,571)}=39.25$; right: $F_{(3,25)}=24.39$, control vs SORLA TG, WT vs SORLA TG, SORLA TG vs SORLA KO, $p<0.0001$, one-way ANOVA with Tukey's multiple comparisons). $\boldsymbol{B}$, $\boldsymbol{E}$, Data are mean \pm SD. ${ }^{* * *} p<0.001 ;{ }^{* * *} p<0.0001$; one-way ANOVA followed by Tukey's multiple comparisons.

transgenic SORLA overexpression can enhance neurite length in hippocampal neurons, and accelerate regenerative neurite extension following mechanical wounding.

\section{sSORLA enhances neurite extension and regeneration}

Ectodomain shedding by a sheddase on the plasma membrane releases an sSORLA fragment comprising most of the SORLA extracellular region (sSORLA), which has been shown to enhance smooth muscle cell migration (Zhu et al., 2004; Jiang et al., 2008). Given that sSORLA can upregulate ERK and Rac1 pathways to affect migratory dynamics (Jiang et al., 2008), we sought to determine whether sSORLA can also promote neurite outgrowth and regeneration. As expected, we found that elevation in SORLA levels concomitantly elevates the release of sSORLA in SORLA TG neurons cultured in vitro (Fig. $2 A$ ), and verified overexpression of SORLA in SORLA TG lysates (Fig. $2 A$, "neuronal lysates"). Given that APP is also cleaved into a soluble form, we assayed sAPP $\alpha$ secretion in WT and SORLA TG neurons to determine whether general enhancement of cell surface cleavage was apparent with SORLA overexpression. Although we observed accumulation of $\operatorname{sAPP} \alpha$ in these cultures, we observed relatively little difference in $\operatorname{SAPP} \alpha$ between cultured WT and SORLA TG neurons (Fig. 2A). To determine whether sSORLA is sufficient to enhance neurite outgrowth, we expressed an

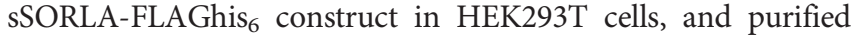
sSORLA from conditioned media (Fig. 2B). Purified sSORLA added to WT DIV3 cultured hippocampal neurons significantly enhanced neurite length (Fig. 2C), as well as axonal length (Fig. 2D). Similar to what we observed in SORLA TG neurons, sSORLA treatment did not significantly affect the distribution of neurite numbers (Fig. 2E).

Next, we determined whether purified sSORLA could enhance neurite regeneration into a wound area. We found that treatment of WT cortical neurons with sSORLA for $16 \mathrm{~h}$ promotes neurite extension into the wound area in a dose-dependent manner (Fig. 3A,B), with significantly increased neurite outgrowth induced by 100 and $150 \mathrm{ng} / \mathrm{ml}$ sSORLA (Fig. 3A,B). In addition, we could recapitulate sSORLA-mediated enhancement of neurite extension using conditioned medium from SORLA TG neurons, while SORLA WT and SORLA KO 
A SORLA TG vs WT cortex, phosphopeptide analysis

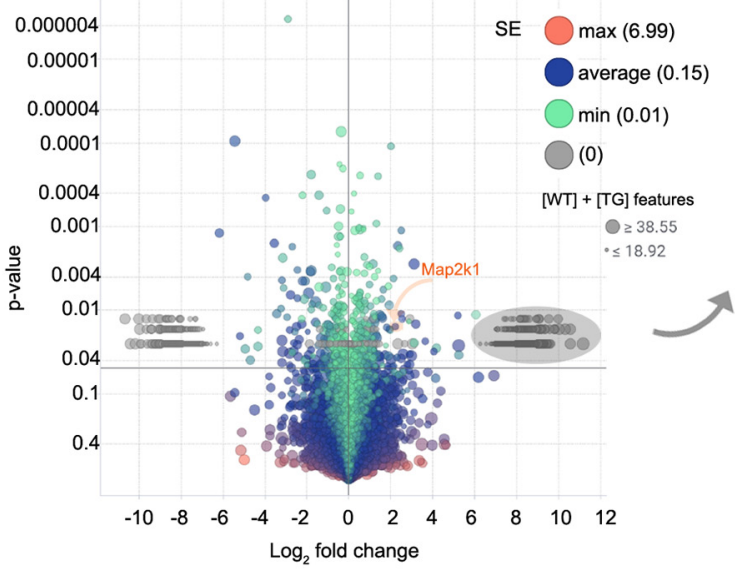

B

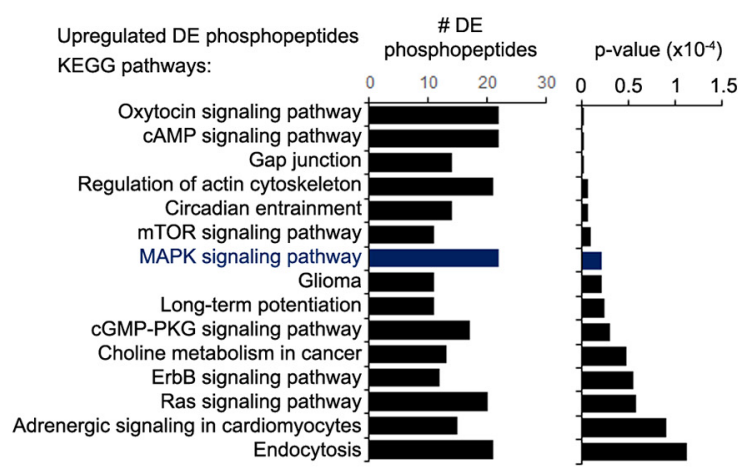

UPregulated SORLA TG-specific phosphopeptides,

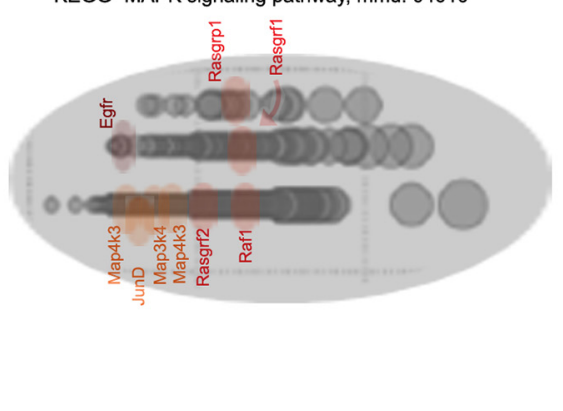

D

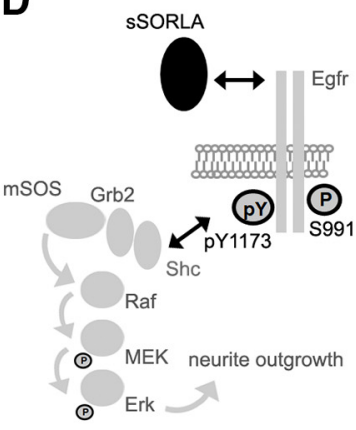

C Upregulated DE phosphopeptides, SORLA TG vs WT KEGG "MAPK signaling pathway, mmu: 04010"

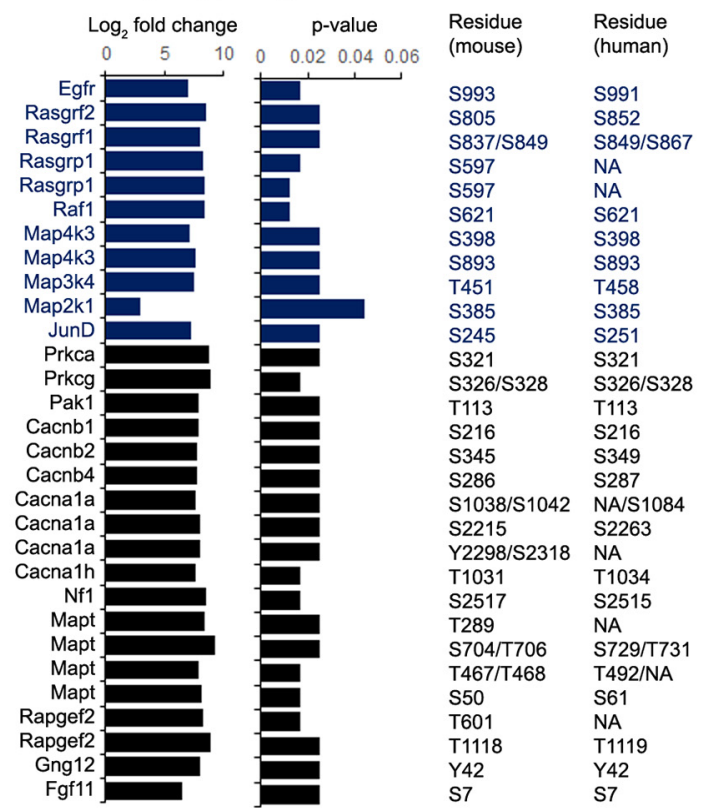

Figure 4. SSORLA triggers activation of the EGFR/ERK pathway in neurons. A, Phosphoproteomic characterization of SORLA TG versus WT mouse cortex. Lysates from 3-month-old SORLA TG and WT cortex ( $n=4$ per genotype) were subjected to phosphoproteomic analysis by mass spectrometry. Differentially enriched phosphopeptides are shown in the volcano plot. Dot sizes indicate relative phosphopeptide abundance (MS1 intensity sum across samples; see Materials and Methods). Color schemes represent statistical test SE between samples as indicated. Phosphopeptides detected exclusively in SORLA TG cortex are shown in the adjacent magnified plot. Components of the KEGG MAPK signaling pathway identified by GO DAVID analysis are indicated in the individual plots, where relevant MAPK signaling components are represented in orange (MAPK pathway components) or red (upstream Ras/MAPK regulators). B, Top 15 KEGG pathways identified from upregulated phosphopeptides in SORLA TG hippocampus. C, Components within the KEGG MAPK signaling pathway with upregulated peptides identified in SORLA TG hippocampus. Components of the canonical EGFR/MAPK pathway are highlighted in blue. Mapped mouse and orthologous human phosphoresidues are shown. $\boldsymbol{D}$, Schematic model depicting putative activation of the MAPK pathway, potentially through SORLA interaction and activation of the EGF receptor. EGFR S991 phosphorylation (mouse S993) was identified through proteomic analysis, and pY residues, such as EGFR pY1173 associated with EGF stimulation and downstream Ras/ERK activation, were identified by immunoblot analysis in response to sSORLA treatment.

conditioned media featured reduced or undetectable sSORLA levels and did not promote neurite extension compared with the control (neurobasal medium only) (Fig. 3C-E). Thus, sSORLA can promote neurite extension and regeneration.

\section{sSORLA triggers activation of the ERK MAPK pathway through EGFR}

To identify potential mediators of sSORLA-dependent neuritogenic enhancement, we compared global phosphoprotein profiles in SORLA TG and WT mouse cortex in search of phosphoregulatory pathways that may be associated with neurite outgrowth. We identified a total of 1423 phosphopeptides that were differentially expressed in SORLA TG brain (736 upregulated, and 687 downregulated; $p<0.05, \log _{2}$ fold change $>2$; Fig. 4A). GO DAVID analysis for KEGG pathways potentially enriched in SORLA TG phosphopeptide datasets revealed enrichment in various phosphoregulatory pathways, including RAS and MAPK, EGFR/ErbB, as well as components related to actin cytoskeleton regulation and endocytosis (Fig. 4B,C).

Given that SORLA has been shown to modulate the activation of cell surface receptors, such as TrkB, ErbB2, EphA4, and the insulin receptor (Rohe et al., 2013; Huang et al., 2017; Pietila et al., 2019), we searched for enrichment of differentially expressed phosphopeptides from cell surface receptors and their intracellular effectors. Interestingly, we observed enrichment of a mouse EGFR phosphopeptide containing phosphorylated S993 (corresponding to human EGFR S991) in SORLA TG brain. This EGFR phosphosite has been previously implicated in receptor endocytosis (Tong et al., 2009). We also observed enrichment of other components in the KEGG MAPK signaling pathway category, including Rasgrf2, Rasgrf1, Rasgrp1, Raf1, Map4k3, Map3k4, Map2k1, and JunD (Fig. 4A-C). Since EGFR S991 phosphorylation is reported to slowly accumulate with EGF 

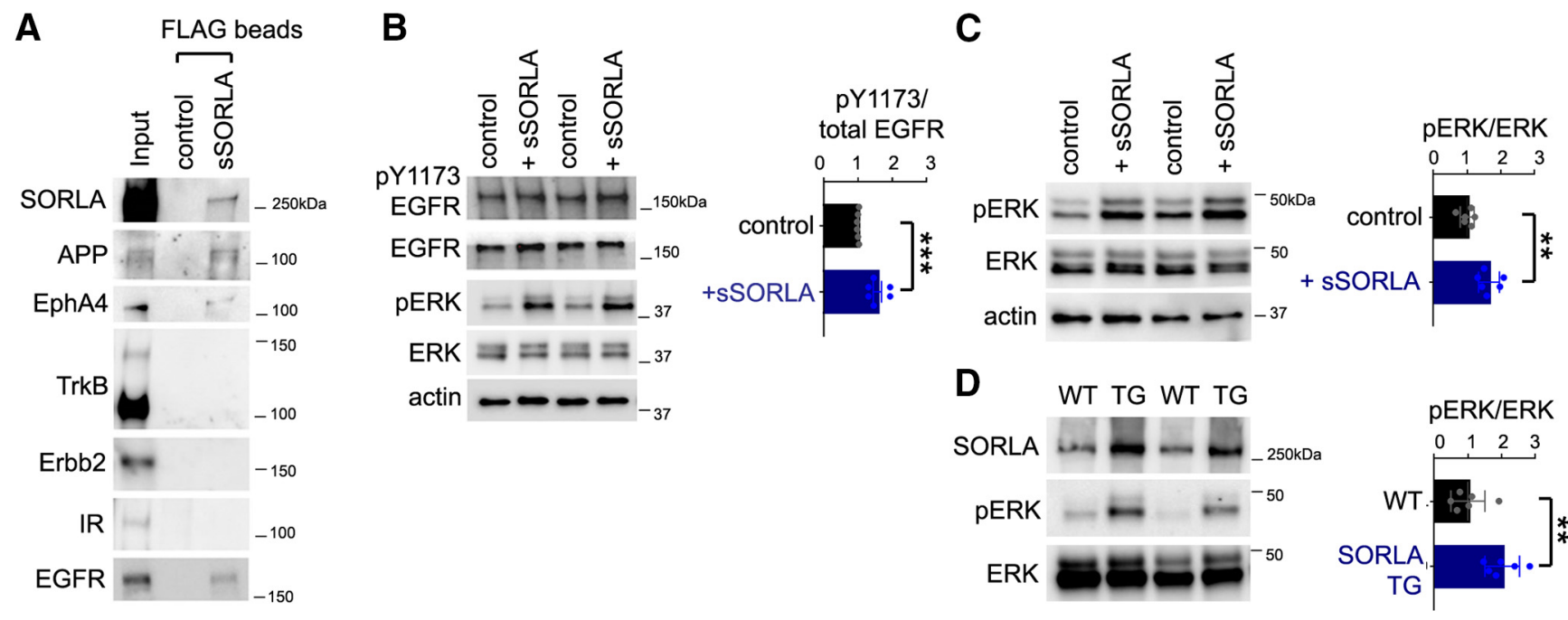

Figure 5. SSORLA triggers EGFR and ERK activation. A, Characterizing SSORLA interaction with cell surface proteins. FLAG beads coupled to sSORLA-FLAGhis (or control FLAG beads) were incubated with WT mouse brain lysate, and associated proteins were detected by immunoblotting as indicated (20 ng lysate input shown). B, Effects of sSORLA on EGFR/ERK activation. Cortical neurons at DIV8 were treated with SSORLA for $0.5 \mathrm{~h}$, and neuronal lysates were immunoblotted for pY1173 EGFR, EGFR, pERK, ERK, or actin as indicated. Densitometric measurements from additional experiments were plotted in the adjacent graph $(p=0.0008, t$ test). C, $\boldsymbol{D}$, sSORLA and SORLA overexpression triggers ERK activation. C, DIV9 cortical neurons were treated with sSORLA for $0.5 \mathrm{~h}$, and neuronal lysates were immunoblotted for pERK, ERK, or actin. pERK levels were quantified by densitometry, and normalized to ERK levels are shown in the adjacent graph (control set to 1.0; 6 replicates from three experiments) $(p=0.0016, t$ test). $\boldsymbol{D}$. Hippocampal lysates from 3.5-month-old WT $(n=6)$ and SORLA TG (TG) $(n=6)$ animals were immunoblotted for SORLA, pERK, or ERK as indicated. pERK and ERK levels were quantified and normalized as in $C\left(p=0.0061, t\right.$ test). Data are mean \pm SE. ${ }^{* *} p<0.01 ;{ }^{* * *} p<0.001$; Student's $t$ test.

stimulation (Olsen et al., 2006) and is predicted to be a potential MAPK phosphorylation site (www.phosphonet.ca), this suggests that sSORLA may trigger downstream MAPK activation by interacting with EGFR and promoting its activation (Fig. 4D). To test this, we immobilized purified sSORLA on FLAG beads and incubated sSORLA-FLAG beads (or FLAG beads alone) with mouse brain lysates. We observed specific coprecipitation of sSORLA with previously characterized interacting proteins, such as APP and EphA4 (Andersen et al., 2006; Rogaeva et al., 2007; Huang et al., 2016), and observed novel interactions between sSORLA and EGFR, which have not been previously described (Fig. 5A).

Although did not detect differences in EGFR tyrosine phosphorylation between WT and SORLA TG cortex, enhanced pS993 phosphorylation suggests that SORLA/sSORLA overexpression increases EGFR activation. To directly examine the effects of sSORLA on EGFR activation (as seen through EGFR tyrosine phosphorylation), we treated cultured primary cortical neurons with purified sSORLA, and examined EGFR phosphorylation by immunoblot analysis. We found that sSORLA treatment enhances EGFR phosphorylation on Y1173, a residue previously shown to be phosphorylated in response to EGF stimulation and consequently mediates docking to the Shc adaptor, thereby recruiting Grb2 to trigger downstream Ras/ERK activation (Batzer et al., 1994; Okabayashi et al., 1994) (Fig. 5B). Indeed, we found that primary cortical neurons treated with sSORLA show increased EGFR pY1173 phosphorylation and concurrent ERK phosphorylation (Fig. 5B,C). Importantly, we also observed ERK activation in response to sSORLA exposure in primary neurons (Fig. 5C), and in SORLA TG hippocampus compared with WT (Fig. 5D) by immunoblot analysis. Together, these results suggest that sSORLA binds EGFR and stimulates activation of a canonical EGFR/ERK pathway.

EGFR and ERK activation are required for sSORLA-induced enhancement of neurite regeneration

Our phosphoproteomic and immunoblot analyses indicate that the EGFR/ERK pathway is upregulated by SORLA overexpression or exposure to sSORLA. Since previous studies have demonstrated that EGFR (Goldshmit et al., 2004) and ERK activation can promote neurite outgrowth (Kim et al., 1997; Perron and Bixby, 1999; Gerecke et al., 2004; Wang et al., 2011; Wu et al., 2012), we examined whether EGFR and ERK activation are essential for sSORLAdependent neurite regeneration. Treatment of primary cortical neurons with the EGFR inhibitor, Tyrphostin (100 $\mu \mathrm{M}$ AG-1478), revealed almost complete inhibition of EGF- or sSORLA-dependent EGFR and ERK activation (Fig. 6A,B). We also measured the effects of EGFR inhibition on neurite outgrowth following wound injury in cortical neurons treated with EGF or sSORLA, which revealed that AG-1478 treatment abrogates the enhancement of neurite outgrowth induced by EGF or sSORLA (Fig. 6C,D).

To determine whether sSORLA-mediated neuritogenic effects are dependent on ERK activation, we treated WT cortical neurons with sSORLA in the presence or absence of the MEK inhibitor, PD98059, and evaluated consequent effects on ERK activation and neurite outgrowth. We found that, while sSORLA significantly elevates pERK levels in cortical neurons, concomitant treatment with PD98059 abrogates ERK activation (Fig. 6E). Furthermore, PD98059 abrogated enhanced neurite regeneration with sSORLA treatment (Fig. $6 F, G$ ). Together, these results indicate that sSORLA stimulates neurite regeneration through activation of EGFR and ERK.

\section{sSORLA and SORLA overexpression activate Fos in brain and cultured neurons}

To identify potential downstream mediators of sSORLA-dependent EGFR/ERK signaling, we performed RNAseq analysis to identify changes in global gene expression in hippocampal tissue from WT and SORLA TG mice. We identified 1379 DEGs in SORLA TG hippocampus compared with WT, with a total of 449 upregulated and 930 downregulated genes (Fig. 7A,B; Extended Data Fig. 7-1). KEGG and GO analyses identified numerous KEGG pathways related to cell signaling, such as "Ras signaling," "MAPK signaling," "focal adhesion" pathways, and "axon guidance" (Fig. 7C; Extended Data Fig. 7-1). Interestingly, 
A

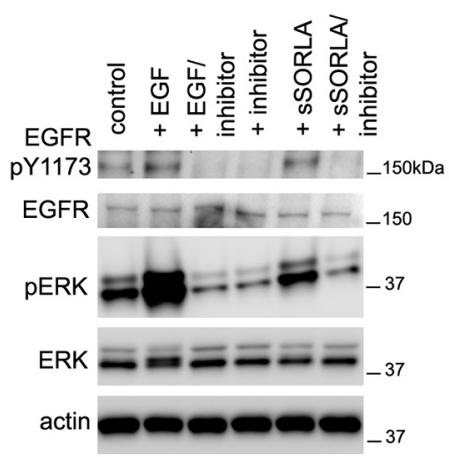

B

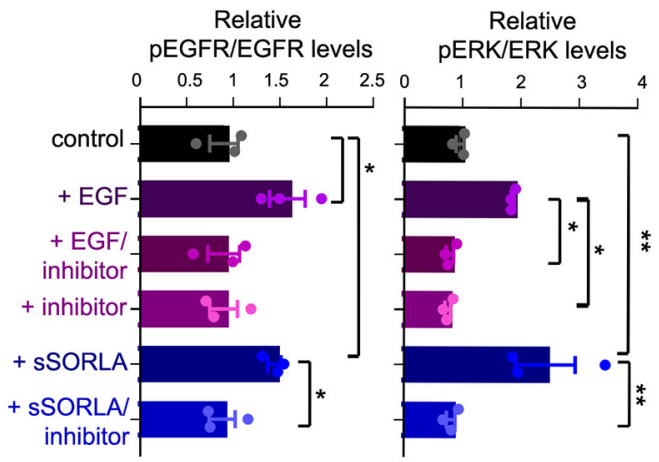

C

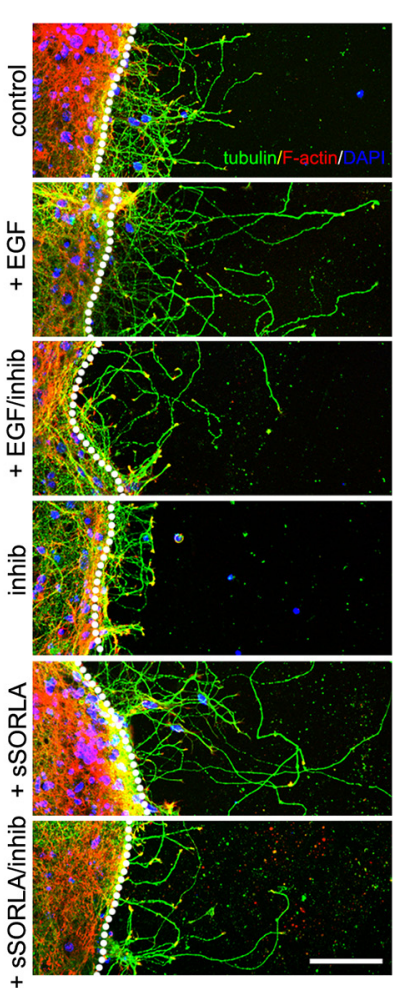

D total neurite length $(\mu \mathrm{m})$

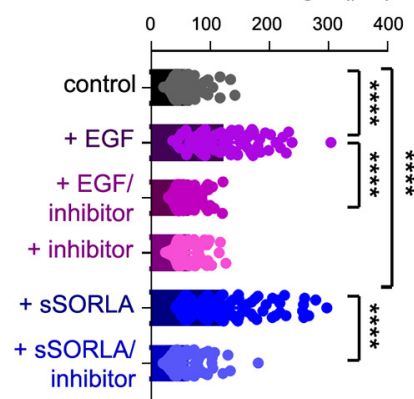

avg neurite length $(\mu \mathrm{m})$

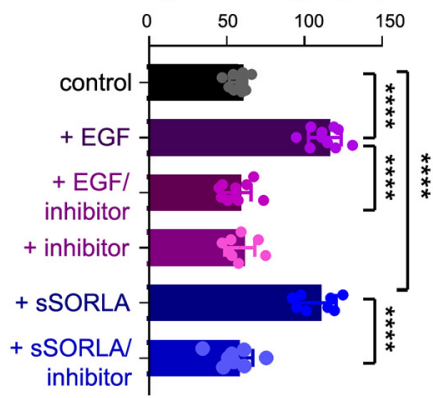

$\mathbf{E}$

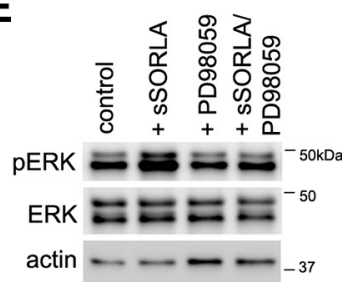

Relative pERK/ERK levels (neurons)

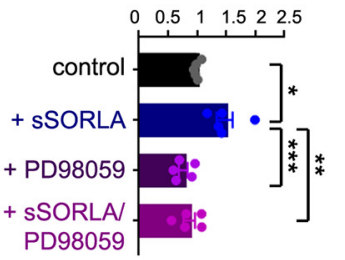

F

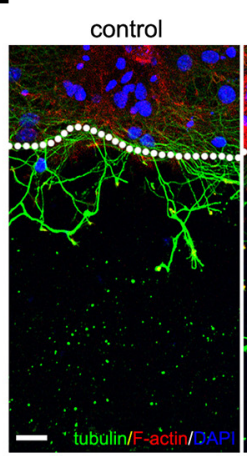

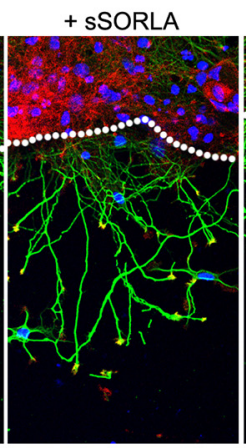
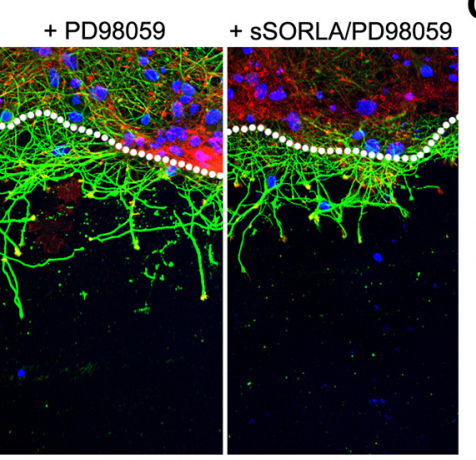

G

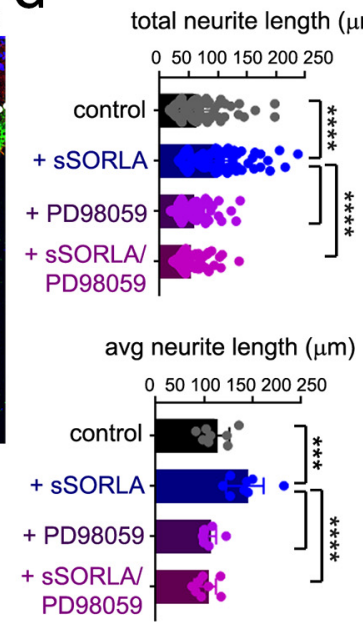

Figure 6. EGFR and ERK activation is required for sSORLA-dependent enhancement of neurite regeneration. EGFR inhibition abrogates EGF and SSORLA-dependent EGFR activation. $\boldsymbol{A}$, Cultured cortical neurons were treated with EGF or SSORLA with or without the EGF inhibitor, AG-1478 as described in Materials and Methods. Lysates were then immunoblotted as indicated. $\boldsymbol{B}$, pEGFR or pERK levels normalized to total EGFR or ERK levels in immunoblots from $\boldsymbol{A}$ were plotted compared with the untreated control (set to 1.0): left, control versus EGF ( $p=0.0497)$, control versus sSORLA $(p=0.0315)$, sSORLA versus sSORLA/inhibitor $(p=0.0228)$, $t$ test; right: $F_{(5,12)}=10.52$, control versus sSORLA $(p=0.0047)$, EGF versus EGF/inhibitor $(p=0.0407)$, EGF versus inhibitor $(p=0.0335)$, sSORLA versus SSORLA/inhibitor ( $p=0.0022)$, one-way ANOVA with Tukey's multiple comparisons. Characterizing the effects of EGFR inhibition on sSORLA-dependent enhancement of neurite regeneration. C, DIV6 cortical neurons were subjected to mechanical wound injury, and treated with EGF or SSORLA in the presence or absence of the EGF inhibitor AG-1478. After $16 \mathrm{~h}$, neurons were fixed and stained for tubulin (green), F-actin (red), and with DAPI (blue). Scale bar, $60 \mu \mathrm{m}$. D, Images from C were quantified for neurite length from the wound border in all neurons (total neurite length, top) or averaged per image (average neurite length, bottom) (control, 201 neurites in 10 imaging fields; EGF, 150 neurites in 10 imaging fields; EGF with inhibitor, 166 neurites in 10 imaging fields; inhibitor, 156 neurites in 8 imaging fields; SSORLA, 177 neurites in 8 imaging fields; sSORLA with inhibitor, 169 neurites in 8 imaging fields; 3 coverslips from 2 independent dissections/3 cultures per treatment) (top: $F_{(5,1014)}=128.9$; bottom: $F_{(5,48)}=69.98$, control vs EGF, control vs SSORLA, EGF vs EGF/inhibitor, SSORLA vs sSORLA/inhibitor, $p<0.0001$; bottom: one-way ANOVA with Tukey's multiple comparisons). $E$, WT DIV7 cortical neurons were treated with SSORLA $(150 \mathrm{ng} / \mathrm{ml})$, the MEK inhibitor PD98059 $(100 \mu \mathrm{m})$, or sSORLA and PD98059 in combination for $16 \mathrm{~h}$. Neuronal lysates were then subjected to immunoblot analysis as indicated, and pERK levels were quantified and normalized to total ERK $\left(F_{(3,16)}=11.62\right.$, control vs sSORLA, $p=0.0104$; 5 SORLA vs PD98059, $p=0.0003$; SSORLA vs SSORLA/PD98059, $p=0.0014$, one-way ANOVA with Tukey's multiple comparisons). $\boldsymbol{F}$, WT DIV7 cortical neurons were subjected to mechanical wounding, and exposed to SSORLA, PD98059, or SSORLA with PD98059 as in E. Neurons were fixed $16 \mathrm{~h}$ following wounding, stained for tubulin (green), F-actin (red), and DAPI (blue), and imaged by confocal microscopy. Scale bar, $20 \mu \mathrm{m}$. $\boldsymbol{G}$, Images in $\boldsymbol{F}$ were quantified for total neurite length (from a minimum of 127 neurons/treatment; top) or averaged neurite length per imaged field (from a minimum of 9 fields/treatment; bottom): control, 180 neurites in 12 images, sSORLA, 140 neurites in 12 images; PD98059, 127 neurites in 11 images; SSORLA with PD98089, 134 neurites in 9 images; 3 coverslips from 2 independent dissections/ 3 cultures per treatment; top: $F_{(5,532)}=50.85$, sSORLA versus control, sSORLA versus PD98059 and sSORLA versus sSORLA/PD98059 ( $\left.p<0.0001\right)$; bottom: $F_{(3,28)}=$ 14 , control versus sSORLA $(p=0.0008)$, sSORLA versus PD98059 and SSORLA versus sSORLA/PD98059 $(p<0.0001)$, one-way ANOVA with Tukey's multiple comparisons. $\boldsymbol{B}, \boldsymbol{E}$, Data are mean \pm SE. $\boldsymbol{D}, \boldsymbol{G}$, Data are mean \pm SD. Statistical significance: $\boldsymbol{B}, \mathrm{pERK} / \mathrm{ERK}, \boldsymbol{D}, \boldsymbol{E}, \boldsymbol{G}$, by One-Way ANOVA followed by Tukey's multiple comparisons; and $\boldsymbol{B}, \mathrm{pEGFR/EGFR,} \mathrm{One-Way} \mathrm{ANOVA}\left(F_{(5,12)}=4.625\right.$, $p=0.0139)$ was used in $(\boldsymbol{B})$, where $p$-values were determined by Student's $t$-test. ${ }^{*} p<0.05,{ }^{* *} p<0.01,{ }^{* * *} p<0.001$, ${ }^{* * *} p<0.0001$. 
A

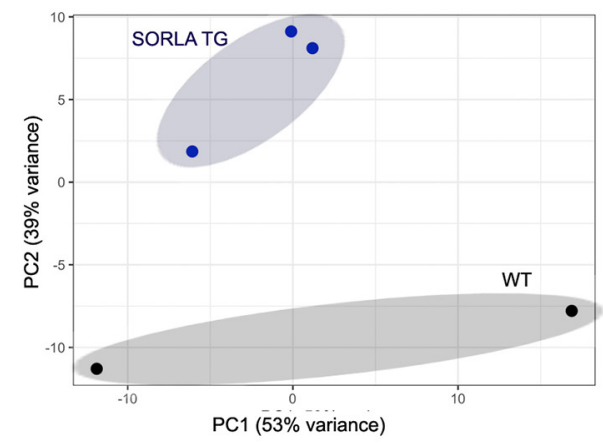

B

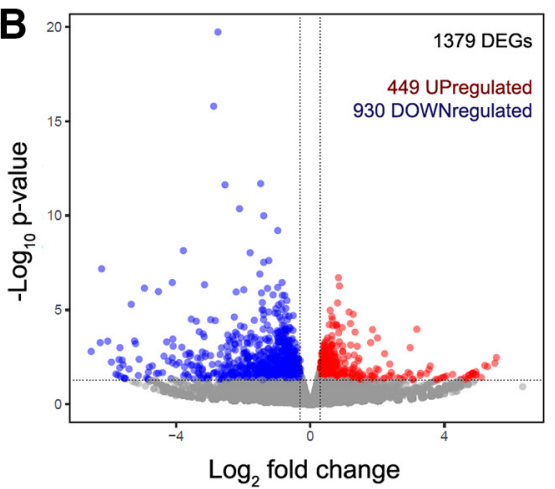

SORLA TG vs WT
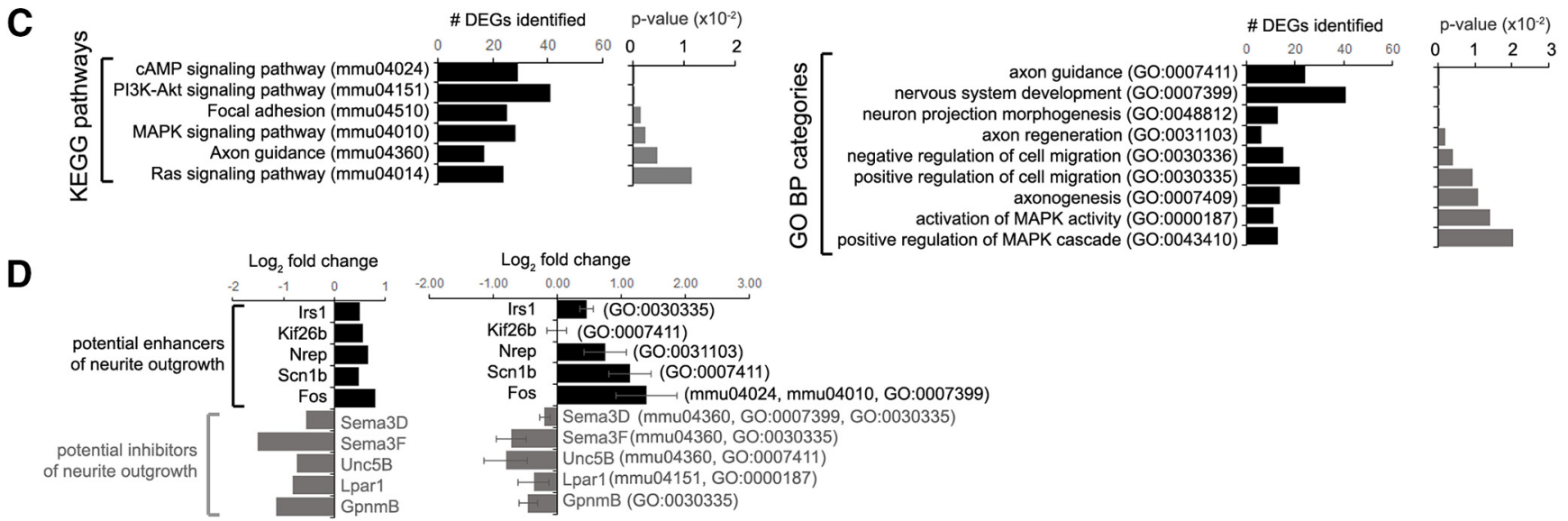

Figure 7. Changes in global mRNA expression in SORLA TG hippocampus. A, PCA analysis of transcriptomic profiles from WT (black) and SORLA TG (blue) hippocampus from 3-month-old mice. $B$, Volcano plot represents differentially expressed transcripts in SORLA TG versus WT hippocampus. A total of 449 significantly upregulated (red) and 930 downregulated (blue) DEGs were identified ( Log $_{2}$ fold change $<-0.3$ or $>0.3, p<0.05$ ). C, Selected KEGG pathways and GO BP categories, enriched in SORLA TG versus WT RNAseq datasets, were identified by GO DAVID analysis. Pathways relevant to signal transduction (KEGG) or neurite outgrowth (GO BP) are shown (see also Extended Data Fig. 7-1). D, Potential enhancers (black) and inhibitors (gray) of neurite outgrowth were selected from the KEGG or G0 BP categories in C for validation by qRT-PCR. RNAseq profiles (left) and qRT-PCR validation (right, mean \pm SE) are shown. The qRTPCR profiles show expected upregulation (enhancers) or downregulation (inhibitors) in the SORLA TG hippocampus, with the exception of Kif26b.

differentially expressed transcripts in SORLA TG hippocampus were also prominently represented in GO Biological Process (BP) categories associated with neuronal morphogenesis, including "neuron projection morphogenesis," "axon regeneration," "axonogenesis," and "activation of MAPK activity" (Fig. 7C).

Next, we filtered individual DEGs within GO BP categories pertaining to axonal/dendritic outgrowth and regeneration, and searched for upregulated potential enhancers of neurite outgrowth, and downregulated potential inhibitors of neurite outgrowth. We identified five upregulated enhancers of neurite outgrowth (Irs1, Kif26b, Nrep, Scn1b, Fos) in our RNAseq dataset, and five downregulated inhibitors of neurite outgrowth (Sema3D, Sema3F, Unc5B, Lpar1, GpnmB) (Fig. 7D), and validated upregulation or downregulation of these transcripts by qRT-PCR analysis, with the sole exception of Kif26b, which showed little or no change (Fig. 7D).

Among the candidate DEGs identified by transcriptomic analysis, we deemed Fos to be particularly relevant to neurite outgrowth and ERK activation. Fos is an immediate early response gene, and together with Jun, forms the AP-1 transcription activation complex (Hai and Curran, 1991; Shaulian and Karin, 2002). Importantly, Fos expression is rapidly induced during neurite regeneration in axotomized neurons (Taylor et al., 2005), and exogenous Fos expression is sufficient to induce neurite outgrowth in the absence of external stimuli, such as NGF (Gil et al., 2004). ERK MAPK signaling has been shown to induce Fos expression through upstream Elk-1 phosphorylation (a "ternary complex factor," or TCF family member) (Janknecht et al., 1993; Janknecht, 1995), which functions coordinately with serum response factors at the upstream Fos serum-response element (Mylona et al., 2011; O’Donnell et al., 2012). Fos is also induced by growth factors, such as NGF, which enhance neurite outgrowth (Gabellini et al., 1992; Gil et al., 2004). Together, this suggests that Fos induction and activation may be triggered in response to sSORLA-dependent ERK activation.

To test the involvement of Fos downstream of SORLA, we examined whether sSORLA treatment and SORLA overexpression affect Fos activation in vitro and in vivo. In agreement with upregulated Fos mRNA levels observed in SORLA TG hippocampus (Fig. 7D), we detected increased nuclear Fos localization in SORLA TG mouse hippocampus (Fig. $8 A$ ). Furthermore, we observed increased Fos protein expression in cultured neurons treated with sSORLA or NGF (Fig. $8 B$ ). Consistent with the increase in nuclear Fos translocation observed in SORLA TG hippocampus, we also detected increased nuclear Fos distribution in cultured neurons treated with sSORLA or NGF (Fig. $8 C, D$ ). Moreover, we found that the MEK inhibitor, PD98059, abrogates nuclear Fos translocation induced by sSORLA treatment (Fig. 8E,F). Together, these results demonstrate a novel role for SORLA and its soluble form, sSORLA in enhancing neurite outgrowth and regeneration through activation of the EGFR/ ERK pathway, and downstream Fos upregulation and nuclear translocation. 
A
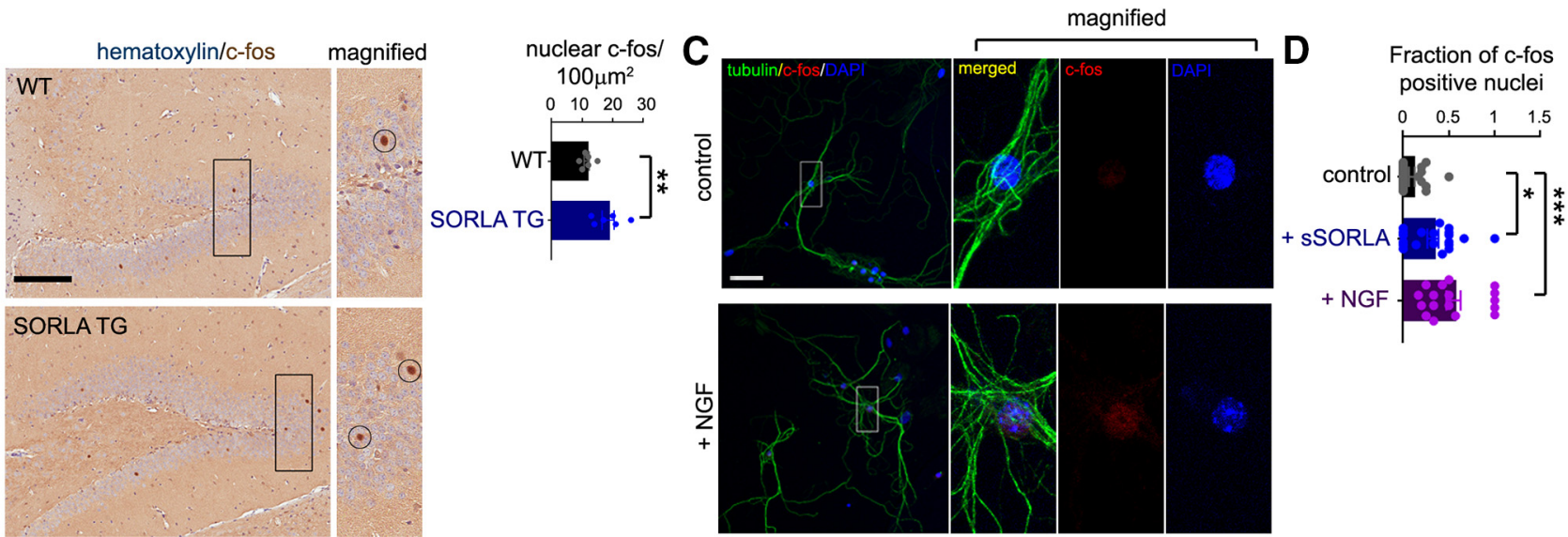

B
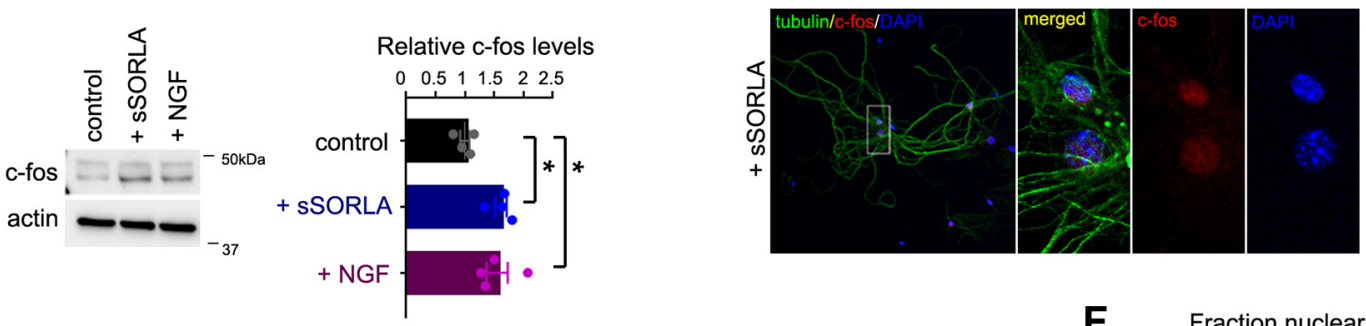

$\mathbf{E}$
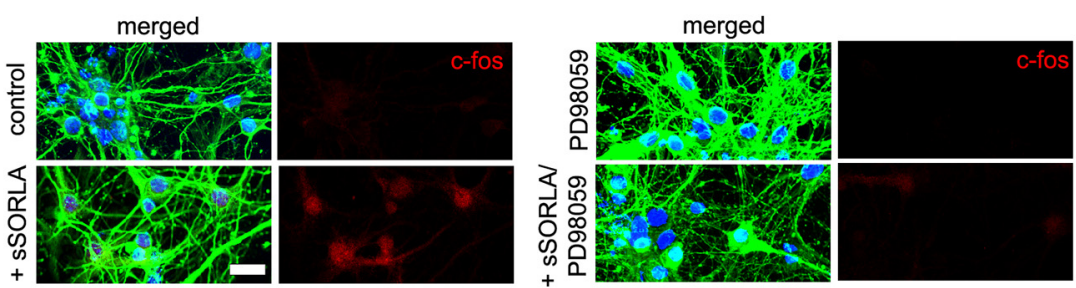

F Fraction nuclear c-fos

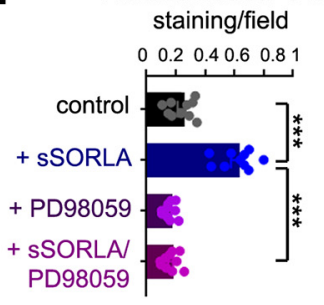

Figure 8. SSORLA and SORLA overexpression triggers Fos upregulation and activation. A, Brain tissue from 4-month-old WT and SORLA TG mice was sectioned, and sagittal sections were stained for nuclei (hematoxylin, blue) or Fos (brown). Scale bar, $150 \mu \mathrm{m}$. Images were obtained from the dentate gyrus region using an Aperio Slide Scanner (Leica Microsystems). Nuclear Fos in $100 \mu \mathrm{m}^{2}$ sections in the dentate gyrus region was scored, and quantification is shown in the adjacent graph ( 3 animals, 6 sections/genotype; male mice were analyzed; $p=0.0087, t$ test). $B$, Fos levels from DIV12 cortical neurons were treated with SSORLA $(150 \mathrm{ng} / \mathrm{ml})$ or NGF $(20 \mathrm{ng} / \mathrm{ml})$ for $60 \mathrm{~min}$, and processed for immunoblot analysis. Quantification of Fos levels normalized to actin from immunoblots is shown in the adjacent graph (compared with controls, set to 1.0): $F_{(2.9)}=6.926$, sSORLA versus control $(p=0.0199)$, NGF versus control $(p=0.0343)$, one-way ANOVA with Tukey's multiple comparisons. C, DIV18 WT hippocampal neurons were treated with SSORLA or NGF, stained for tubulin (green), Fos (red), or DAPI, and visualized by confocal microscopy. Scale bar, $60 \mu \mathrm{m}$. D, Fraction of neurons from the experiments in $\mathbf{C}$ with nuclear Fos staining were scored. Data points represent Fos nuclei/total nuclei (control, 49 neurons in 19 images; SSORLA, 55 neurons in 18 images; NGF, 71 neurons in 20 images; 5 coverslips from 2 independent dissections/3 cultures per treatment): $F_{(2.55)}=15.47$, control versus $s 5 O R L A$ $(p=0.0138)$, control versus NGF $(p<0.0001)$, one-way ANOVA with Tukey's multiple comparisons. $E$, WT cortical neurons at DIV8 were treated with sSORLA, PD98059, or sSORLA/PD98059 together for $16 \mathrm{~h}$, and neurons were fixed and stained for tubulin (green), Fos (red), and DAPI (blue). Scale bar, $20 \mu \mathrm{m}$. $\boldsymbol{F}$, Neurons showing nuclear Fos staining over total neurons per field were quantified from images in $\boldsymbol{E}$ (control, 283 neurites in 11 images; sSORLA, 259 neurites in 11 images; PD98059, 334 neurites in 11 images; sSORLA with PD98059, 317 neurites in 11 images; 3 coverslips from 2 independent dissections/3 cultures per treatment): $F_{(3,36)}=72.83$, control versus sSORLA and sSORLA versus sSORLA/PD98059 ( $\left.p<0.0001\right)$, one-way ANOVA followed by Tukey's multiple comparisons. Graphs represent mean \pm SE. A, Student's $t$ test. $\boldsymbol{B}, \boldsymbol{D}, \boldsymbol{F}$, One-way ANOVA followed by Tukey's multiple comparisons. ${ }^{*} p<0.05,{ }^{* *} p<0.01,{ }^{* * *} p<0.001$.

\section{Discussion}

Although SORLA ectodomain shedding had been previously characterized for well over a decade in mammalian cells (Hermey et al., 2006), human neural precursor and neuroendocrine cell lines (Hampe et al., 2000), and invertebrate Hydra species, such as Chlorohydra viridissima (Hampe et al., 1999), how sSORLA potentially affects neuronal morphogenesis and function has not yet been explored. Importantly, SORLA has been previously implicated as a genetic risk factor for $\mathrm{AD}$; recent evidence identified significant enrichment of rare loss-of-function variants of the SORL1 gene in AD (Raghavan et al., 2018), suggesting that SORLA dysregulation could significantly promote $\mathrm{AD}$ onset and/or pathogenesis. Interestingly, analysis of cerebrospinal fluid from $\mathrm{AD}$ patients indicates that sSORLA levels are reduced in $\mathrm{AD}$, which correlates with a reduction in SAPP forms in cerebrospinal fluid ( $\mathrm{Ma}$ et al., 2009). Further, release of sSORLA has been shown to be induced in acute mouse brain slices (i.e., brain slice injury) (Hampe et al., 2000), and with regenerative wounding stimulus in Hydra (Hampe et al., 1999), suggesting that sSORLA levels increase in response to neuronal injury. Although this provides evidence that sSORLA levels are altered pathologically, whether sSORLA can affect brain function has remained unclear. The prevailing assumption has been that SORLA function is mediated primarily by intact, cell-associated SORLA in neurons. Until now, whether sSORLA can be released from neurons and its capacity to alter neuronal function are issues that have not been addressed.

Here, we describe a new role for sSORLA in enhancing neurite outgrowth and regeneration through activation of the EGFR/ ERK signaling pathway. The findings described here for sSORLA are intriguingly similar to observations with $\alpha$-secretase-cleaved $\operatorname{sAPP} \alpha$. Purified sAPP $\alpha$ has been previously shown to enhance neurite outgrowth in cortical neurons (Araki et al., 1991), potentially through interactions with the $\beta 1$ integrin receptor 
(Young-Pearse et al., 2008), and by enhancing or potentiating neurite outgrowth mediated by NGF-induced signaling (Milward et al., 1992; Wallace et al., 1997). Similar to sSORLA, neuronal exposure to $\operatorname{sAPP} \alpha$ also enhances neurite outgrowth through ERK activation (Gakhar-Koppole et al., 2008; Chasseigneaux et al., 2011). In contrast to sSORLA, however, acute exposure to $\operatorname{sAPP} \alpha$ was not found to detectably affect ERK phosphorylation/activation directly (Gakhar-Koppole et al., 2008). Thus, ADAM protease-dependent cleavage of $\mathrm{AD}$-related transmembrane proteins, such as SORLA and APP, may serve to promote ERK-dependent neuritogenesis through autocrine or paracrine sSORLA/sAPP $\alpha$ pathways.

Although neurite outgrowth and neuritogenic activity are clearly critical during the development of the CNS, how neuritogenic soluble proteins, such as sSORLA and $\operatorname{sAPP} \alpha$, may be involved in late-stage $\mathrm{AD}$ is less clear. Histologic studies have detected neurite/axonal outgrowth in brain regions with synaptic pathology (Masliah et al., 1991), suggesting that pathogenic events may be associated with compensatory neurite regeneration. Although neurite growth is critical during early brain development, neurite extension is also important during neurogenesis in the adult dentate gyrus within the hippocampal formation. Generation of nascent neurons from neural progenitor cells within the dentate gyrus is thought to be important for synaptic plasticity, pattern separation (Sahay et al., 2011), and spatial memory (Stone et al., 2011). Although alterations in neurogenesis have been reported in some $\mathrm{AD}$ mouse models $\mathrm{Mu}$ and Gage, 2011) as well as human AD patients (Jin et al., 2004; Crews et al., 2010; Perry et al., 2012; Gomez-Nicola et al., 2014), whether neurogenesis can attenuate $\mathrm{AD}$ pathogenesis and memory impairment remains unclear. Interestingly, recent evidence indicates that memory in $\mathrm{AD}$ mice can be improved through combined enhancement of neurogenesis and stimulation of BDNF pathways (Choi et al., 2018). Given that BDNF is a neurotrophic factor that enhances neurite outgrowth (Cheng et al., 2011; Wang et al., 2015), together with evidence that BDNF can enhance SORLA expression (Rohe et al., 2009; Young et al., 2015), it may be interesting in future studies to determine whether BDNF-associated effects on memory enhancement may be dependent, at least in part, on SORLA/sSORLA. Indeed, neurotrophins and neurotrophin receptors are currently targets of interest in AD therapy (Cao et al., 2018); whether SORLA/ sSORLA association with neurotrophin pathways can be of future interest remains to be determined.

Our results demonstrate a role for SSORLA in the regeneration of dendrites and axons, which suggests that SORLA/sSORLA may be important in pathologically relevant contexts, such as nerve regeneration following injury. Interestingly, expression of the SORLA homolog in the Hydrozoan, Chlorohydra viridissima is enriched in developing buds, representing sites of reproductive outgrowth (Hampe et al., 1999), suggesting that SORLA plays a likely role in development and regeneration. Murine SORLA shows high expression in the brain of developing embryos (Hermans-Borgmeyer et al., 1998), suggesting that SORLA may also be important in early neuronal differentiation and morphogenesis. Although a role for SORLA as a neuritogenic modifier has not been previously demonstrated, our results integrate well into broader known functions of SORLA in cell growth, differentiation, and morphogenesis as described in Hydra and mice. Moreover, observations that SORLA is expressed in the spinal cord (Jacobsen et al., 1996) suggest that sSORLA could play a role in promoting axon regeneration following spinal cord injury. Indeed, neurotrophic BDNF infusion or grafting BDNF-secreting cells in axotomized rodent spinal cord injury models have demonstrated neuroprotective effects, including enhancement of axon fiber regeneration (Bregman et al., 1997; Ye and Houle, 1997), neuronal survival (Kobayashi et al., 1997; Tobias et al., 2003; Ruitenberg et al., 2004; Kwon et al., 2007), and promoting neuronal plasticity during recovery from spinal cord injury (Weishaupt et al., 2012). Furthermore, sSORLA infusion may potentially circumvent toxicity effects associated with BDNF. For example, BDNF secretion by activated microglia may complicate recovery by promoting neuropathic pain pathways (Coull et al., 2005; Zhang et al., 2012), and aggravate spasticity by inducing overexcitability in spinal cord motor neurons (Boyce et al., 2012; Lu et al., 2012). Thus, potential downstream mediators of nerve recovery, such as sSORLA, may be particularly beneficial in ameliorating nervous system injury and neurodegenerative events.

\section{References}

Andersen OM, Schmidt V, Spoelgen R, Gliemann J, Behlke J, Galatis D, McKinstry WJ, Parker MW, Masters CL, Hyman BT, Cappai R, Willnow TE (2006) Molecular dissection of the interaction between amyloid precursor protein and its neuronal trafficking receptor SorLA/LR11. Biochemistry 45:2618-2628.

Andersen OM, Reiche J, Schmidt V, Gotthardt M, Spoelgen R, Behlke J, von Arnim CA, Breiderhoff T, Jansen P, Wu X, Bales KR, Cappai R, Masters CL, Gliemann J, Mufson EJ, Hyman BT, Paul SM, Nykjaer A, Willnow TE (2005) Neuronal sorting protein-related receptor sorLA/LR11 regulates processing of the amyloid precursor protein. Proc Natl Acad Sci USA 102:13461-13466.

Araki W, Kitaguchi N, Tokushima Y, Ishii K, Aratake H, Shimohama S, Nakamura S, Kimura J (1991) Trophic effect of beta-amyloid precursor protein on cerebral cortical neurons in culture. Biochem Biophys Res Commun 181:265-271.

Batzer AG, Rotin D, Urena JM, Skolnik EY, Schlessinger J (1994) Hierarchy of binding sites for Grb2 and Shc on the epidermal growth factor receptor. Mol Cell Biol 14:5192-5201.

Boyce VS, Park J, Gage FH, Mendell LM (2012) Differential effects of brainderived neurotrophic factor and neurotrophin-3 on hindlimb function in paraplegic rats. Eur J Neurosci 35:221-232.

Bregman BS, McAtee M, Dai HN, Kuhn PL (1997) Neurotrophic factors increase axonal growth after spinal cord injury and transplantation in the adult rat. Exp Neurol 148:475-494.

Caglayan S, Takagi-Niidome S, Liao F, Carlo AS, Schmidt V, Burgert T, Kitago Y, Fuchtbauer EM, Fuchtbauer A, Holtzman DM, Takagi J, Willnow TE (2014) Lysosomal sorting of amyloid-beta by the SORLA receptor is impaired by a familial Alzheimer's disease mutation. Sci Transl Med 6:223ra20.

Cao J, Hou J, Ping J, Cai D (2018) Advances in developing novel therapeutic strategies for Alzheimer's disease. Mol Neurodegener 13:64.

Chasseigneaux S, Dinc L, Rose C, Chabret C, Coulpier F, Topilko P, Mauger G, Allinquant B (2011) Secreted amyloid precursor protein beta and secreted amyloid precursor protein alpha induce axon outgrowth in vitro through Egr1 signaling pathway. PLoS One 6:e16301.

Cheng PL, Song AH, Wong YH, Wang S, Zhang X, Poo MM (2011) Selfamplifying autocrine actions of BDNF in axon development. Proc Natl Acad Sci USA 108:18430-18435.

Choi SH, Bylykbashi E, Chatila ZK, Lee SW, Pulli B, Clemenson GD, Kim E, Rompala A, Oram MK, Asselin C, Aronson J, Zhang C, Miller SJ, Lesinski A, Chen JW, Kim DY, van Praag H, Spiegelman BM, Gage FH, Tanzi RE (2018) Combined adult neurogenesis and BDNF mimic exercise effects on cognition in an Alzheimer's mouse model. Science 361: eaan8821.

Coull JA, Beggs S, Boudreau D, Boivin D, Tsuda M, Inoue K, Gravel C, Salter MW, De Koninck Y (2005) BDNF from microglia causes the shift in neuronal anion gradient underlying neuropathic pain. Nature 438:10171021.

Crews L, Adame A, Patrick C, Delaney A, Pham E, Rockenstein E, Hansen L, Masliah E (2010) Increased BMP6 levels in the brains of Alzheimer's disease patients and APP transgenic mice are accompanied by impaired neurogenesis. J Neurosci 30:12252-12262.

Fjorback AW, Seaman M, Gustafsen C, Mehmedbasic A, Gokool S, Wu C, Militz D, Schmidt V, Madsen P, Nyengaard JR, Willnow TE, Christensen 
EI, Mobley WB, Nykjær A, Andersen OM (2012) Retromer binds the FANSHY sorting motif in SorLA to regulate amyloid precursor protein sorting and processing. J Neurosci 32:1467-1480.

Fu AK, Hung KW, Huang H, Gu S, Shen Y, Cheng EY, Ip FC, Huang X, Fu WY, Ip NY (2014) Blockade of EphA4 signaling ameliorates hippocampal synaptic dysfunctions in mouse models of Alzheimer's disease. Proc Natl Acad Sci USA 111:9959-9964.

Gabellini N, Minozzi MC, Leon A, Dal Toso R (1992) Nerve growth factor transcriptional control of c-fos promoter transfected in cultured spinal sensory neurons. J Cell Biol 118:131-138.

Gakhar-Koppole N, Hundeshagen P, Mandl C, Weyer SW, Allinquant B, Müller U, Ciccolini F (2008) Activity requires soluble amyloid precursor protein alpha to promote neurite outgrowth in neural stem cell-derived neurons via activation of the MAPK pathway. Eur J Neurosci 28:871882.

Gerecke KM, Wyss JM, Carroll SL (2004) Neuregulin-1beta induces neurite extension and arborization in cultured hippocampal neurons. Mol Cell Neurosci 27:379-393.

Gil GA, Bussolino DF, Portal MM, Alfonso Pecchio A, Renner ML, Borioli GA, Guido ME, Caputto BL (2004) c-Fos activated phospholipid synthesis is required for neurite elongation in differentiating PC12 cells. Mol Biol Cell 15:1881-1894.

Goldshmit Y, Walters CE, Scott HJ, Greenhalgh CJ, Turnley AM (2004) SOCS2 induces neurite outgrowth by regulation of epidermal growth factor receptor activation. J Biol Chem 279:16349-16355.

Gomez-Nicola D, Suzzi S, Vargas-Caballero M, Fransen NL, Al-Malki H, Cebrian-Silla A, Garcia-Verdugo JM, Riecken K, Fehse B, Perry VH (2014) Temporal dynamics of hippocampal neurogenesis in chronic neurodegeneration. Brain 137:2312-2328.

Hai T, Curran T (1991) Cross-family dimerization of transcription factors Fos/Jun and ATF/CREB alters DNA binding specificity. Proc Natl Acad Sci USA 88:3720-3724.

Hampe W, Urny J, Franke I, Hoffmeister-Ullerich SA, Herrmann D, Petersen CM, Lohmann J, Schaller HC (1999) A head-activator binding protein is present in hydra in a soluble and a membrane-anchored form. Development 126:4077-4086.

Hampe W, Riedel IB, Lintzel J, Bader CO, Franke I, Schaller HC (2000) Ectodomain shedding, translocation and synthesis of SorLA are stimulated by its ligand head activator. J Cell Sci 113:4475-4485.

Hermans-Borgmeyer I, Hampe W, Schinke B, Methner A, Nykjaer A, Susens U, Fenger U, Herbarth B, Schaller HC (1998) Unique expression pattern of a novel mosaic receptor in the developing cerebral cortex. Mech Dev 70:65-76.

Hermey G, Sjogaard SS, Petersen CM, Nykjaer A, Gliemann J (2006) Tumour necrosis factor alpha-converting enzyme mediates ectodomain shedding of Vps10p-domain receptor family members. Biochem J 395:285-293.

Huang da W, Sherman BT, Lempicki RA (2009a) Systematic and integrative analysis of large gene lists using DAVID bioinformatics resources. Nat Protoc 4:44-57.

Huang da W, Sherman BT, Lempicki RA (2009b) Bioinformatics enrichment tools: paths toward the comprehensive functional analysis of large gene lists. Nucleic Acids Res 37:1-13.

Huang TY, Zhao Y, Li X, Wang X, Tseng IC, Thompson R, Tu S, Willnow TE, Zhang YW, Xu H (2016) SNX27 and SORLA interact to reduce amyloidogenic subcellular distribution and processing of amyloid precursor protein. J Neurosci 36:7996-8011.

Huang TY, Zhao Y, Jiang LL, Li X, Liu Y, Sun Y, Pina-Crespo JC, Zhu B, Masliah E, Willnow TE, Pasquale EB, Xu H (2017) SORLA attenuates EphA4 signaling and amyloid beta-induced neurodegeneration. J Exp Med 214:3669-3685.

Jacobsen L, Madsen P, Moestrup SK, Lund AH, Tommerup N, Nykjaer A, Sottrup-Jensen L, Gliemann J, Petersen CM (1996) Molecular characterization of a novel human hybrid-type receptor that binds the alpha2-macroglobulin receptor-associated protein. J Biol Chem 271:31379-31383.

Janknecht R (1995) Regulation of the c-fos promoter. Immunobiology 193:137-142.

Janknecht R, Ernst WH, Pingoud V, Nordheim A (1993) Activation of ternary complex factor Elk-1 by MAP kinases. EMBO J 12:5097-5104.

Jiang M, Bujo H, Zhu Y, Yamazaki H, Hirayama S, Kanaki T, Shibasaki M, Takahashi K, Schneider WJ, Saito Y (2006) Pitavastatin attenuates the
PDGF-induced LR11/uPA receptor-mediated migration of smooth muscle cells. Biochem Biophys Res Commun 348:1367-1377.

Jiang M, Bujo H, Ohwaki K, Unoki H, Yamazaki H, Kanaki T, Shibasaki M, Azuma K, Harigaya K, Schneider WJ, Saito Y (2008) Ang II-stimulated migration of vascular smooth muscle cells is dependent on LR11 in mice. J Clin Invest 118:2733-2746.

Jin K, Peel AL, Mao XO, Xie L, Cottrell BA, Henshall DC, Greenberg DA (2004) Increased hippocampal neurogenesis in Alzheimer's disease. Proc Natl Acad Sci USA 101:343-347.

Kim B, Leventhal PS, Saltiel AR, Feldman EL (1997) Insulin-like growth factor-I-mediated neurite outgrowth in vitro requires mitogen-activated protein kinase activation. J Biol Chem 272:21268-21273.

Kobayashi NR, Fan DP, Giehl KM, Bedard AM, Wiegand SJ, Tetzlaff W (1997) BDNF and NT-4/5 prevent atrophy of rat rubrospinal neurons after cervical axotomy, stimulate GAP-43 and Talpha1-tubulin mRNA expression, and promote axonal regeneration. J Neurosci 17:9583-9595.

Kwon BK, Liu J, Lam C, Plunet W, Oschipok LW, Hauswirth W, Di Polo A, Blesch A, Tetzlaff W (2007) Brain-derived neurotrophic factor gene transfer with adeno-associated viral and lentiviral vectors prevents rubrospinal neuronal atrophy and stimulates regeneration-associated gene expression after acute cervical spinal cord injury. Spine (Phila Pa 1976) 32:1164-1173.

Lambert JC, Ibrahim-Verbaas CA, Harold D, Naj AC, Sims R, Bellenguez C, Jun G, DeStefano AL, Bis JC, Beecham GW, Grenier-Boley B, Russo G, Thornton-Wells TA, Jones N, Smith AV, Chouraki V, Thomas C, Ikram MA, Zelenika D, Vardarajan BN, et al. (2013) Meta-analysis of 74,046 individuals identifies 11 new susceptibility loci for Alzheimer's disease. Nat Genet 45:1452-1458.

Lu P, Blesch A, Graham L, Wang Y, Samara R, Banos K, Haringer V, Havton L, Weishaupt N, Bennett D, Fouad K, Tuszynski MH (2012) Motor axonal regeneration after partial and complete spinal cord transection. J Neurosci 32:8208-8218.

Ma QL, Galasko DR, Ringman JM, Vinters HV, Edland SD, Pomakian J, Ubeda OJ, Rosario ER, Teter B, Frautschy SA, Cole GM (2009) Reduction of SorLA/LR11, a sorting protein limiting beta-amyloid production, in Alzheimer disease cerebrospinal fluid. Arch Neurol 66:448457.

Masliah E, Mallory M, Hansen L, Alford M, Albright T, DeTeresa R, Terry R, Baudier J, Saitoh T (1991) Patterns of aberrant sprouting in Alzheimer's disease. Neuron 6:729-739.

Milward EA, Papadopoulos R, Fuller SJ, Moir RD, Small D, Beyreuther K, Masters CL (1992) The amyloid protein precursor of Alzheimer's disease is a mediator of the effects of nerve growth factor on neurite outgrowth. Neuron 9:129-137.

Miyashita A, Koike A, Jun G, Wang LS, Takahashi S, Matsubara E, Kawarabayashi T, Shoji M, Tomita N, Arai H, Asada T, Harigaya Y, Ikeda M, Amari M, Hanyu H, Higuchi S, Ikeuchi T, Nishizawa M, Suga M, Kawase Y, et al. (2013) SORL1 is genetically associated with late-onset Alzheimer's disease in Japanese, Koreans and Caucasians. PLoS One 8: e58618.

Mu Y, Gage FH (2011) Adult hippocampal neurogenesis and its role in Alzheimer's disease. Mol Neurodegener 6:85.

Mylona A, Nicolas R, Maurice D, Sargent M, Tuil D, Daegelen D, Treisman $\mathrm{R}$, Costello $\mathrm{P}$ (2011) The essential function for serum response factor in T-cell development reflects its specific coupling to extracellular signalregulated kinase signaling. Mol Cell Biol 31:267-276.

O'Donnell A, Odrowaz Z, Sharrocks AD (2012) Immediate-early gene activation by the MAPK pathways: what do and don't we know? Biochem Soc Trans 40:58-66.

Okabayashi Y, Kido Y, Okutani T, Sugimoto Y, Sakaguchi K, Kasuga M (1994) Tyrosines 1148 and 1173 of activated human epidermal growth factor receptors are binding sites of Shc in intact cells. J Biol Chem 269:18674-18678

Olsen JV, Blagoev B, Gnad F, Macek B, Kumar C, Mortensen P, Mann M (2006) Global, in vivo, and site-specific phosphorylation dynamics in signaling networks. Cell 127:635-648.

Perron JC, Bixby JL (1999) Distinct neurite outgrowth signaling pathways converge on ERK activation. Mol Cell Neurosci 13:362-378.

Perry EK, Johnson M, Ekonomou A, Perry RH, Ballard C, Attems J (2012) Neurogenic abnormalities in Alzheimer's disease differ between stages of neurogenesis and are partly related to cholinergic pathology. Neurobiol Dis $47: 155-162$. 
Pietila M, Sahgal P, Peuhu E, Jantti NZ, Paatero I, Narva E, Al-Akhrass H, Lilja J, Georgiadou M, Andersen OM, Padzik A, Sihto H, Joensuu H, Blomqvist M, Saarinen I, Bostrom PJ, Taimen P, Ivaska J (2019) SORLA regulates endosomal trafficking and oncogenic fitness of HER2. Nat Commun 10:2340.

Raghavan NS, Brickman AM, Andrews H, Manly JJ, Schupf N, Lantigua R, Wolock CJ, Kamalakaran S, Petrovski S, Tosto G, Vardarajan BN, Goldstein DB, Mayeux R, Alzheimer's Disease Sequencing Project (2018) Whole-exome sequencing in 20,197 persons for rare variants in Alzheimer's disease. Ann Clin Transl Neurol 5:832-842.

Richter M, Murai KK, Bourgin C, Pak DT, Pasquale EB (2007) The EphA4 receptor regulates neuronal morphology through SPAR-mediated inactivation of Rap GTPases. J Neurosci 27:14205-14215.

Rogaeva E, Meng Y, Lee JH, Gu Y, Kawarai T, Zou F, Katayama T, Baldwin CT, Cheng R, Hasegawa H, Chen F, Shibata N, Lunetta KL, PardossiPiquard R, Bohm C, Wakutani Y, Cupples LA, Cuenco KT, Green RC, Pinessi L, et al. (2007) The neuronal sortilin-related receptor SORL1 is genetically associated with Alzheimer disease. Nat Genet 39:168-177.

Rohe M, Hartl D, Fjorback AN, Klose J, Willnow TE (2013) SORLA-mediated trafficking of $\operatorname{TrkB}$ enhances the response of neurons to BDNF. PLoS One 8:e72164.

Rohe M, Synowitz M, Glass R, Paul SM, Nykjaer A, Willnow TE (2009) Brain-derived neurotrophic factor reduces amyloidogenic processing through control of SORLA gene expression. J Neurosci 29:15472-15478.

Ruitenberg MJ, Blits B, Dijkhuizen PA, te Beek ET, Bakker A, van Heerikhuize JJ, Pool CW, Hermens WT, Boer GJ, Verhaagen J (2004) Adeno-associated viral vector-mediated gene transfer of brain-derived neurotrophic factor reverses atrophy of rubrospinal neurons following both acute and chronic spinal cord injury. Neurobiol Dis 15:394-406.

Sahay A, Scobie KN, Hill AS, O'Carroll CM, Kheirbek MA, Burghardt NS, Fenton AA, Dranovsky A, Hen R (2011) Increasing adult hippocampal neurogenesis is sufficient to improve pattern separation. Nature 472:466470.

Scherzer CR, Offe K, Gearing M, Rees HD, Fang G, Heilman CJ, Schaller C, Bujo H, Levey AI, Lah JJ (2004) Loss of apolipoprotein E receptor LR11 in Alzheimer disease. Arch Neurol 61:1200-1205.

Schmidt V, Baum K, Lao A, Rateitschak K, Schmitz Y, Teichmann A, Wiesner B, Petersen CM, Nykjaer A, Wolf J, Wolkenhauer O, Willnow TE (2012) Quantitative modelling of amyloidogenic processing and its influence by SORLA in Alzheimer's disease. EMBO J 31:187-200.

Schmidt V, Schulz N, Yan X, Schurmann A, Kempa S, Kern M, Bluher M, Poy MN, Olivecrona G, Willnow TE (2016) SORLA facilitates insulin receptor signaling in adipocytes and exacerbates obesity. J Clin Invest 126:2706-2720.

Shaulian E, Karin M (2002) AP-1 as a regulator of cell life and death. Nat Cell Biol 4:E131-E136.

Spoelgen R, von Arnim CA, Thomas AV, Peltan ID, Koker M, Deng A, Irizarry MC, Andersen OM, Willnow TE, Hyman BT (2006) Interaction of the cytosolic domains of sorLA/LR11 with the amyloid precursor protein (APP) and beta-secretase beta-site APP-cleaving enzyme. J Neurosci 26:418-428.

Stone SS, Teixeira CM, Devito LM, Zaslavsky K, Josselyn SA, Lozano AM, Frankland PW (2011) Stimulation of entorhinal cortex promotes adult neurogenesis and facilitates spatial memory. J Neurosci 31:13469-13484.
Taylor AM, Blurton-Jones M, Rhee SW, Cribbs DH, Cotman CW, Jeon NL (2005) A microfluidic culture platform for CNS axonal injury, regeneration and transport. Nat Methods 2:599-605.

Tobias CA, Shumsky JS, Shibata M, Tuszynski MH, Fischer I, Tessler A, Murray M (2003) Delayed grafting of BDNF and NT-3 producing fibroblasts into the injured spinal cord stimulates sprouting, partially rescues axotomized red nucleus neurons from loss and atrophy, and provides limited regeneration. Exp Neurol 184:97-113.

Tong J, Taylor P, Peterman SM, Prakash A, Moran MF (2009) Epidermal growth factor receptor phosphorylation sites Ser991 and Tyr998 are implicated in the regulation of receptor endocytosis and phosphorylations at Ser1039 and Thr1041. Mol Cell Proteomics 8:2131-2144.

Wallace WC, Akar CA, Lyons WE (1997) Amyloid precursor protein potentiates the neurotrophic activity of NGF. Brain Res Mol Brain Res 52:201212 .

Wang L, Chang X, She L, Xu D, Huang W, Poo MM (2015) Autocrine action of BDNF on dendrite development of adult-born hippocampal neurons. J Neurosci 35:8384-8393

Wang X, Wang Z, Yao Y, Li J, Zhang X, Li C, Cheng Y, Ding G, Liu L, Ding $Z$ (2011) Essential role of ERK activation in neurite outgrowth induced by alpha-lipoic acid. Biochim Biophys Acta 1813:827-838.

Weishaupt N, Blesch A, Fouad K (2012) BDNF: the career of a multifaceted neurotrophin in spinal cord injury. Exp Neurol 238:254-264.

Whittle AJ, Jiang M, Peirce V, Relat J, Virtue S, Ebinuma H, Fukamachi I, Yamaguchi T, Takahashi M, Murano T, Tatsuno I, Takeuchi M, Nakaseko C, Jin W, Jin Z, Campbell M, Schneider WJ, Vidal-Puig A, Bujo H (2015) Soluble LR11/SorLA represses thermogenesis in adipose tissue and correlates with BMI in humans. Nat Commun 6:8951.

Wu CL, Chou YH, Chang YJ, Teng NY, Hsu HL, Chen L (2012) Interplay between cell migration and neurite outgrowth determines SH2B1betaenhanced neurite regeneration of differentiated PC12 cells. PLoS One 7: e34999.

Ye JH, Houle JD (1997) Treatment of the chronically injured spinal cord with neurotrophic factors can promote axonal regeneration from supraspinal neurons. Exp Neurol 143:70-81.

Young-Pearse TL, Chen AC, Chang R, Marquez C, Selkoe DJ (2008) Secreted APP regulates the function of full-length APP in neurite outgrowth through interaction with integrin betal. Neural Dev 3:15.

Young JE, Boulanger-Weill J, Williams DA, Woodruff G, Buen F, Revilla AC, Herrera C, Israel MA, Yuan SH, Edland SD, Goldstein LS (2015) Elucidating molecular phenotypes caused by the SORL1 Alzheimer's disease genetic risk factor using human induced pluripotent stem cells. Cell Stem Cell 16:373-385

Zhang X, Xu Y, Wang J, Zhou Q, Pu S, Jiang W, Du D (2012) The effect of intrathecal administration of glial activation inhibitors on dorsal horn BDNF overexpression and hind paw mechanical allodynia in spinal nerve ligated rats. J Neural Transm (Vienna) 119:329-336.

Zhu Y, Bujo H, Yamazaki H, Ohwaki K, Jiang M, Hirayama S, Kanaki T, Shibasaki M, Takahashi K, Schneider WJ, Saito Y (2004) LR11, an LDL receptor gene family member, is a novel regulator of smooth muscle cell migration. Circ Res 94:752-758. 